\title{
Emerging role of m6A methylation modification in ovarian cancer
}

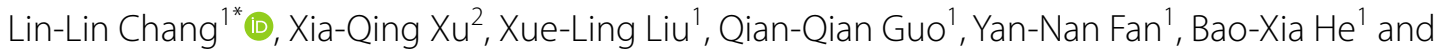 \\ Wen-Zhou Zhang ${ }^{1 *}$
}

\begin{abstract}
m6A (N6-methyladenosine) methylation, a well-known modification in tumour epigenetics, dynamically and reversibly fine tunes the entire process of RNA metabolism. Aberrant levels of m6A and its regulators, which can predict the survival and outcomes of cancer patients, are involved in tumorigenesis, metastasis and resistance. Ovarian cancer (OC) ranks first among gynaecological tumours in the causes of death. At first diagnosis, patients with OC are usually at advanced stages owing to a lack of early biomarkers and effective targets. After treatment, patients with OC often develop drug resistance. This article reviews the recent experimental advances in understanding the role of m6 $\mathrm{A}$ modification in OC, raising the possibility to treat m6A modification and its regulators as promising diagnostic markers and therapeutic targets for OC.
\end{abstract}

Keywords: m6A modification, Methyltransferase, Demethylase, RNA binding protein, OC

\section{Graphical Abstract}

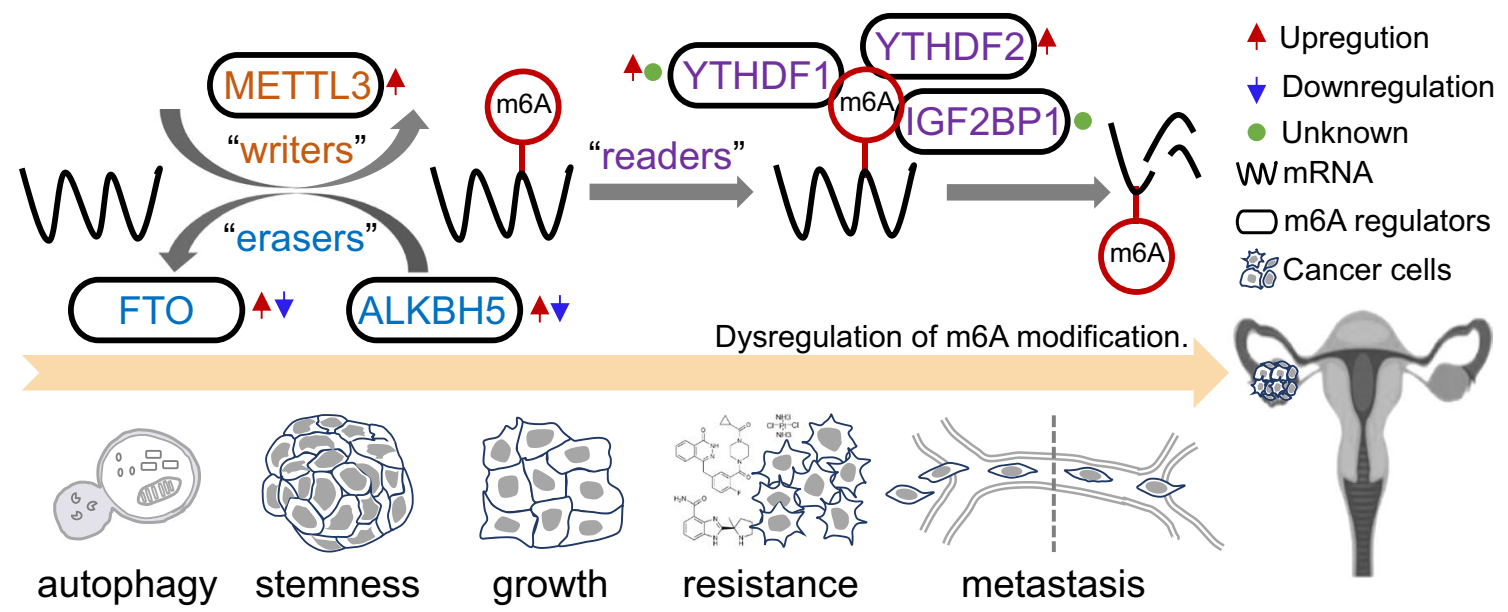

*Correspondence: zlyychanglinlin4398@zzu.edu.cn; zlyyzhangwenzhou0551@zzu.edu.cn

${ }^{1}$ Department of Pharmacy, Affiliated Tumour Hospital of Zhengzhou

University, Henan Cancer Hospital, 127\# Dongming Rd, Zhengzhou 450008, Henan, China

Full list of author information is available at the end of the article

\section{Background}

Ovarian cancer $(\mathrm{OC})$ is the leading death among gynaecological tumours, attracting the attention of researchers [1-3]. According to its histopathology, OC consists of sex cord-stromal tumours, germ cell tumours and epithelial original author(s) and the source, provide a link to the Creative Commons licence, and indicate if changes were made. The images or other third party material in this article are included in the article's Creative Commons licence, unless indicated otherwise in a credit line to the material. If material is not included in the article's Creative Commons licence and your intended use is not permitted by statutory regulation or exceeds the permitted use, you will need to obtain permission directly from the copyright holder. To view a copy of this licence, visit http://creativecommons.org/licenses/by/4.0/. The Creative Commons Public Domain Dedication waiver (http://creativeco mmons.org/publicdomain/zero/1.0/) applies to the data made available in this article, unless otherwise stated in a credit line to the data. 
ovarian cancer (EOC), the last of which accounts for $85 \%$ of OC cases and is associated with an unimproved 5-year survival rate (40\%) [4]. Furthermore, 90\% of EOCs are identified as serous ovarian cancers, which exhibit a poor 10-year survival rate (26\%) [5-7].

One of the hot research topics in OC is early detection and prediction. In addition to imaging, liver function and complete blood count, tumour markers [usually cancer antigen 125 (CA-125)] can assist in primary diagnosis and progression evaluation, and their assessment is minimally invasive $[8,9]$. A concentration of $22 \mathrm{U} / \mathrm{mL}$ CA-125 can detect $66.5 \%$ (95\% CI 49.5-58.4) of ovarian cancers, and an increase in the cut-off to $35 \mathrm{U} / \mathrm{mL}$ allows detection of 41.3\% (95\% CI 33.5-49.5) [8, 9]. However, the concentration of serum CA-125 is controversial because fixed CA-125 cut-offs show no mortality benefit in the general population [9]. Moreover, the AURELIA trial and MRCOV05 trial results indicated that CA-125 was not suitable for surveillance, shown by the lack of an obvious correlation with survival [10]. Thus, there is a lack of efficient detective biomarkers, contributing to $\sim 80 \%$ OC patients diagnosed at metastatic advancedstage, most of which will develop resistance to current clinical therapies $[11,12]$. For advanced OC patients, the first-line treatment usually is surgical cytoreduction and subsequent adjuvant chemotherapy (paclitaxel and carboplatin) and then maintenance therapy [inhibitors of poly (ADP-ribose) polymerase (PARPis), bevacizumab or their combination] [13]. Although the paclitaxel and platinum regimen has been the standard adjuvant chemotherapy over the last 30 years, nearly all patients will endure relapse within 2 years, despite achieving complete remission [10, 13]. PARPis as maintenance monotherapy in SOLO-1 trials displayed promising outcomes for patients with $B R C A$ mutation and/or genomic instability, termed as homologous recombination deficiency (HRD) $[14,15]$. Niraparib also showed a survival benefit (vs. placebo) for the overall population in the PRIMA trial, extending patient survival regardless of $B R C A$ mutation and indicating other possible targets for $O C$ treatment [16]. Cumulative studies have mainly focused on exploring the front-line therapy regimen, neoadjuvant systemic therapy and maintenance therapy, whereas clinical trials have brought no significant improvement in overall survival for OC patients [17]. Referring to immunotherapy, checkpoint inhibitors have poor efficacy because most OCs are characterized by low levels of neoantigens on cancer cells [17]. In summary, OC is still incurable [1719]. Therefore, a greater understanding of the signalling cascades involved in OC progression to identify markers for surveillance detection and targets for new therapies will aid in diagnosis and treatment, which are vital to clinical outcomes for OC patients.
N6-methyladenosine (m6A), a posttranscriptional modification in RNA, is dynamical and reversible [20, 21]. It was discovered in the 1970s, providing a potential avenue for epigenetic studies and a focus for disease-related researches [22-24]. Aberrant m6A levels are observed in many pathological processes, including spermatogenesis, adipogenesis, heat shock response, circadian rhythm and $\mathrm{T}$ cell homeostasis [24]. During porcine spermatogenesis, m6A in transcripts mediates SET domain bifurcated histone lysine methyltransferase 1 (SETDB1) and forkhead box O3 (FOXO3) expression in a timely manner [25]. In response to heat shock, m6A modification on the $5^{\prime}$ untranslated terminal region (UTR) of heat shock protein family $\mathrm{H}$ member 1 (HSPH1) can be protected by the reader YTH domain containing 1 (YTHDC1) to initiate cap-independent translation; m6A modification in GGAAU of lncRNA HSATIII can be sequestered by the reader YTHDC1 to repress m6A-dependent splicing [26-28]. Before adaptive immune initiation, loss of m6A modification in mRNAs of suppressor of cytokine signalling (SOCS) family genes stabilizes the mRNA and enhances the protein expression of targets, finally maintains the survival of naïve T cells [29]. Meanwhile, accumulated evidence shows that dysregulation of m6A levels and $\mathrm{m} 6 \mathrm{~A}$ regulatory proteins are also closely correlated with the progression of multiple tumours [26, 30-33]. As the first discovered methyltransferase, methyltransferaselike 3 (METTL3) accelerates growth of acute myeloid leukaemia (AML) cells via increasing m6A modification within the leukaemia-associated mRNA transcript presenting CCAAT enhancer binding protein zeta (CEBPZ) protein at transcriptional starting sites [34]. Likewise, in most OC cases, METTL3 plays as an oncogene by boosting invasion, migration and proliferation of $\mathrm{OC}$ cells, during which METTL3 targets different types of RNA, including pri-miRNA 126-5p, lncRNA RHPN1 antisense RNA 1 (head to head) (RHPN1-AS1) and mRNA AXL receptor tyrosine kinase $(A X L)$ [35-37]. alkB homologue 5, RNA demethylase (ALKBH5), an m6A demethylase, can orchestrate m6A levels in the $3^{\prime} \mathrm{UTR}$ of programmed cell death-1 ligand-1 (PD-L1) mRNA, stabilize PD-L1 expression in intrahepatic cholangiocarcinoma (ICC), thus sensitize tumour cells to anti-PD1 immunotherapy [38]. There is also evidence for drug resistance modulation by ALKBH5 in OC [39]. Nie reported that upregulated ALKBH5 in OC decodes the m6A modification of Janus kinase $2(J A K 2)$ and stabilizes JAK2 mRNA, subsequently contributing to cisplatin resistance, which is part of the standard adjuvant chemotherapy regimen [41]. Moreover, a recent study reported that YTH N6-methyladenosine RNA binding protein 1 (YTHDF1), an m6A 'reader', enhanced the overall translational output to fuel $\mathrm{OC}$ tumorigenesis and metastasis by recognizing 
m6A-modified eukaryotic translation initiation factor 3 subunit C (EIF3C) mRNA [40]. Collectively, numerous studies have underscored the importance of m6A signalling cascades in distinct cancer types, including $\mathrm{OC}$.

This review will focus on emerging roles of m6A modification in OC. With this summary of new insights, this review not only improves our understanding of m6A signalling cascades but also provides potential markers for early screening for $\mathrm{OC}$ and relapse prediction, thus shedding light upon new strategies to target OC.

\section{Molecular basis for m6A modification}

m6A functions throughout the entire process of RNA metabolism, which includes transport, translation, splicing and transcription, resulting in RNA stability and degradation $[41,42]$. In global cellular RNAs, m6A nearly modifies $50 \%$ of the total methylated ribonucleotide [43, 44]. m6A modification ultimately converges on m6A-related regulators, including 'writers' (methyltransferases), 'erasers' (demethylases) and 'readers', which recognize substrates and show a clear preference for RRACH sequences $(\mathrm{R}=\mathrm{A}$ or $\mathrm{G}$, and $\mathrm{H}=\mathrm{A}$ or $\mathrm{C}$ or $\mathrm{U})[45,46]$. m6A mainly deposites on the $3^{\prime}$ untranslated region $\left(3^{\prime} \mathrm{UTR}\right)$ and within the internal long exon of mRNA [47]. The 5'UTR m6A has also been observed and has been linked to selective eIF3-dependent and eIF4Eindependent translation [48].

The writers include WTAP (Wilms tumour 1-associated protein), METTL3, METTL14 (methyltransferaselike 14), METTL16 (methyltransferase-like 16), RBM15 (RNA binding motif protein 15), KIAA1429 (vir-like m6A methyltransferase associated, VIRMA) and ZC3H13 (zinc finger $\mathrm{CCCH}$ domain-containing protein 13) and catalyse the formation of m6A [49]. The METTL3METTL14 heterodimer takes part in most m6A modifications [50, 51]. Although it possesses no enzymatic methylation activity, WTAP is necessary for the WTAPMETTL3-METTL14 complex to function during the methylation process [52]. Each subunit of this complex possesses completely different catalytic activities and has very distinct roles during the methylation process. METTL3 exerts catalytic activity via adopting methyl group from S-adenosylmethionine (SAM), METTL14 mainly accounts for substrate recognition, and WTAP assists in directing the complex to nuclear spots [52-54].

m6A-modified mRNA is erased by ALKBH5 or fat mass and obesity-associated protein (FTO), yielding decreased total m6A levels in multiple cells $[49,55,56]$. ALKBH5 and FTO, members of the ALKB family, use $\mathrm{Fe}^{2+}$ and 2-oxoglutarate (2OG) as cofactors to decode the m6A modification [55, 57, 58]. Although several studies have unveiled the ability of FTO to erase N6,2' O-dimethyladenosine (m6Am), most substrate of FTO is still the m6A modification, as the total amount of m6A is dominant in divergent cells [59-61]. FTO is extensively expressed in human different kinds of tissues, indicating its pivotal role in energy metabolism [62]. The indispensable interaction between ALKBH5 and DDX3, the latter of which belongs to the family of DEAD-box RNA helicases, may further dictate the essential role of ALKBH5 in RNA metabolism [63].

Currently identified m6A 'readers' include YTH domain family members (YTHDC1, YTHDC2, YTHDF1, YTHDF2 and YTHDF3), heterogeneous nuclear ribonucleoprotein (HNRNP) family members (HNRNPC and HNRNPA2B1), insulin-like growth factor 2 mRNA-binding proteins (IGF2BP1, IGF2BP2 and IGF2BP3), fragile $\mathrm{X}$ mental retardation 1 (FMR1) and leucine rich pentatricopeptide repeat containing (LRPPRC) [48, 64]. These 'readers' possess the ability to recognize m6A modifications in RNAs and generate functional signals [65]. For example, YTHDFs were shown to influence the stability and translation of mRNA [66-69]. YTHDC1 partially mediates splicing events [70-72]. YTHDF1 interacts with m6A-modified mRNA to enhance translation [69, 73, 74]. Through recruiting carbon catabolite repression 4-negative on TATA-less deadenylase complex (CCR4NOT), YTHDF2 destabilizes and further decays the target mRNA $[68,75]$. Taking the diverse types of RNAs into consideration, many more efforts have been made to discover $\mathrm{m} 6 \mathrm{~A}$ readers and unravel the underlying mechanisms.

\section{Biological functions of $\mathrm{m} 6 \mathrm{~A}$ related regulators in ovarian cancer}

Similar to other tumours, the levels of RNA m6A modification are also dynamically modulated by these three regulator types in OC. Abnormal expression of m6A regulators predicts poor prognosis of $\mathrm{OC}$ patients and is involved in proliferation, invasion, metastasis and resistance via m6A-dependent and m6A-independent activity in OC $[37,39,40,76-82]$. Figure 1 shows the pathological roles and underlying mechanisms of m6A regulators in $\mathrm{OC}$ and will be discussed below.

\section{m6A methylation 'writers'}

Although, at present, seven methyltransferases have been discovered, and all OC studies referring to m6A modification research mainly focus on METTL3 [35-37, 76, 83]. Accumulating evidence has revealed that METTL3 overexpression is extensively observed in OC tissues and predicts dismal prognosis. Both in vitro models and in vivo models, loss- and gain-of-function experiments have highlighted the importance of METTL3 during OC progression [35-37, 76, 83] (Table 1). In most other cancer types, METTL3 functions as an oncogene, including 


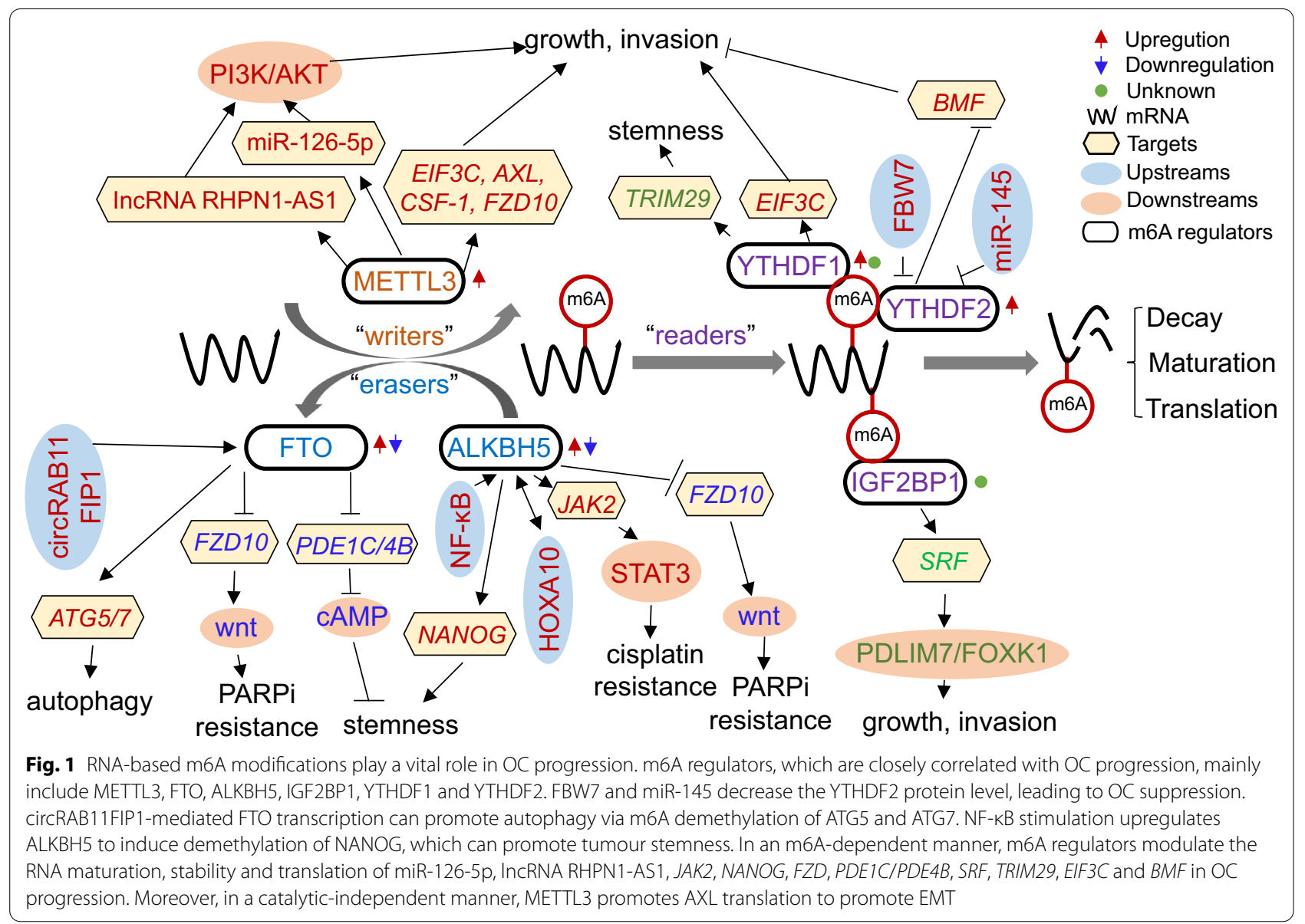

Table 1 The functions of m6A methyltransferases in OC

\begin{tabular}{|c|c|c|c|c|c|}
\hline Writer & Expression & Function & Mechanism & Model & Ref. \\
\hline METTL3 & Up & Promotion & $\begin{array}{l}\text { METTL3 methylates pri-miR-126-5p to promote miR-126-5p maturation, leading to the } \\
\text { activation of PTEN-mediated PI3K/Akt/mTOR pathway }\end{array}$ & In vitro; in vivo & [36] \\
\hline METTL3 & Up & Promotion & METTL3 increases m6A levels of IncRNA RHPN1-AS1 and contributes to its stability & In vitro & [35] \\
\hline METTL3 & Up & Promotion & METTL3 promotes the translation of AXL catalytic-independently & In vitro; in vivo & [37] \\
\hline METTL3 & Up & Promotion & $\begin{array}{l}\text { Independently of METTI14 and WTAP, METTL3 enhances m6A modification in the mRNA of } \\
\text { oncogenes in OC, including AXL, CSF-1, EIF3C and FZD10 }\end{array}$ & In vitro & [76] \\
\hline METTL3 & Up & Promotion & METTL3 knockdown downregulates p-AKT and the downstream effector Cyclin D1 & In vitro & [83] \\
\hline
\end{tabular}

AML, breast cancer, colorectal cancer, gastric cancer, liver cancer, lung cancer, pancreatic cancer and prostate cancer. In several studies, METTL3 has also been found to possess the ability to suppress tumour progression, demonstrated by the higher expression of METTL3 in non-tumour tissues (vs. cancerous tissues) of renal cell carcinoma (RCC) and by the stronger proliferation in mutant METTL3 (vs. wild METTL3) of bladder cancer cells [84-86]. Researchers have identified several types of RNA as targets of METTL3 in OC, including pri-miRNA 126-5p, lncRNA RHPN1-AS1 and mRNA $A X L$, except rRNA and U6 snRNA, which is consistent with previous data indicating that METTL3 is not sensitive to any RNA structural context in vitro $[46,87,88]$. Scientists have also underscored the catalytic-independent activities of METTL3 that contribute to epithelial-mesenchymal transition (EMT) process in OC. A similar functioning mode was found in lung cancer cells demonstrating that METTL3 could recruit translation initiation factors (for example eIF3h) to augment the translation of oncogenes 
(for example $T A Z$ ), independent of its catalytic activity $[89,90]$. However, what stimulates METTL3 overexpression in OC is still unclear, and there is a lack of experimental data inferring the interaction mode between METTL3 and the tumour microenvironment.

In endometrioid EOC, using a dot plot, $\mathrm{Ma}$ et al. showed increased global m6A levels compared to levels in adjacent tissues [76]. Further immunohistochemistry (IHC), western blot (WB) and real-time quantitative polymerase chain reaction (qRT-PCR) data revealed outstanding expression of METTL3 among METTL3, METTL14 and WTAP. In other studies, IHC data also displayed higher levels of METTL3 in OC cancerous tissue compared with the para-cancerous tissue [37, 83]. Liang et al. and Hua et al. both demonstrated that METTL3 was significantly correlated with tumour grade and TNM status, which was consistent with the data in endometrioid EOC verified by Ma et al. [37, 76, 83]. In xenograft mouse models, Hua et al. demonstrated that stable transfection with METTL3 accelerated tumour growth in OVCR-3 cells and that shMETTL3 significantly decreased tumour growth in SK-OV-3 cells in mice [37]. Present data indicates that METTL3 is an oncogene in OC. These preclinical and clinical data for METTL3 not only shed light on the possibility of treating METTL3 as a new prognostic factor for OC progression but also have encouraged scientists to explore the underlying molecular mechanisms to develop new targeting strategies for OC treatment.

m6A modification retunes the destiny of RNA, including maturation and stability [91]. Bi et al. found METTL3 controlled the m6A level of pri-miR-126-5p to accelerate its maturation, which could activate PI3K/AKT/ mTOR signalling [36]. In addition, METTL3 knockdown decreased products of miR-126-5p, and further rescued miR-126-5p-induced proliferation, migration, invasion, and apoptosis inhibition in SK-OV-3 cells [36]. Furthermore, in a xenograft model, silencing METTL3 slowed tumour growth, whereas blockade of phosphatase and tensin homolog (PTEN) counteracted the anticancer effects of shMETTL3 [36]. These data suggest that METTL3 can execute its oncogenic role by activating PTEN/PI3K/AKT signalling in OC. Similarly, Liang et al. reported that METTL3 knockdown impeded OC cells proliferation and invasion, due to interference with AKT signalling cascades [83]. Through RNA immunoprecipitation (RIP) analysis in HEY cells (an EOC cell line), a recent study reported that shMETTL3 resulted in impaired m6A modification in total RNAs including lncRNA RHPN1-AS1, accompanied by accelerated decay of lncRNA RHPN1-AS1, which ultimately promoted tumorigenesis and metastasis of ovarian cancer in vitro [35]. The preliminary results presented in that study not only underpin the protumour roles of METTL3 during OC progression, but also identify different types of RNA as its targets, highlighting an emerging strategy to target mRNA.

Stable complexes are the main gateway to epigenetic methylation machinery. In addition to the KIAA1429/ WTAP complex, one known complex of m6A methyltransferases consists of WTAP, METTL3 and METTL14 $[53,64]$. Although it forms a protein complex to coordinate with WTAP/METTL14 in m6A modification, METTL3 seems at be the core of this process in endometrioid EOC, stemming from the fact that knockdown of METTL3, but not WTAP/METTL14, decreased the m6A levels of associated targets (determined in vitro by RIP-qPCR) [76]. Consistent with these previous data, RIP and WB assays showed that WTAP and METTL14 failed to occupy the transcripts of associated genes and upregulate the levels of associated proteins in OC cells (including TOV-112D and CRL-11731D) [76]. These results are contradictory to the observations from Liu et al. that METTL3, METTL14 and WTAP knockdown in HeLa cells led to decreased m6A in RNA by $\sim 30 \%, \sim 40 \%$ and $\sim 50 \%$, respectively [87]. This may be explained by differences between cell lines and different tumours, which highlights the necessity of exploring the functions of $\mathrm{m} 6 \mathrm{~A}$ regulators in $\mathrm{OC}$ as well as in other tumours. Ma et al. partially verified that METTL3 mediated m6A modification of targets to exert an oncogenic role in endometrioid EOC [76]. In addition, by employing the SRAMP tool, m6A modification located at the $3^{\prime} \mathrm{UTR}$ region of targets was significantly associated with $\mathrm{OC}$, which is the common target site of METTL3 [76]. Another study demonstrated that METTL3 stimulates AXL translation to promote EMT independent of its catalytic activity in OC [37]. In this work, WB analysis showed that the catalytic mutant METTL3 augmented AXL protein levels to the same levels observed with wild-type METTL3 in OVCR-3 cells, underscoring the catalytic-independent role of METTL3 in OC [37], which will yield the possibility to define METTL3 as a 'moonlighting protein' [92]. However, there lack in vivo models to distinguish between catalytic-dependent and catalytic-independent functions of METTL3, specifically the knock-in alleles mice model. Afterall, it becomes increasingly clear that catalytic-independent functions also endow METTL3 with oncogenic roles in OC in addition to its catalyticdependent function. Overall, these studies demonstrate that it may not be sufficient to inhibit only the catalytic activity in future strategies to target METTL3.

\section{m6A methylation 'erasers'}

In relation to human noncancer research, FTO possesses the ability to promote fat formation and obesity, and 
ALKBH5 participates in spermatogenesis, trophoblast invasion and ossification [93, 94]. In OC, dysregulation of ALKBH5 and FTO takes part in proliferation, apoptosis, migration, drug resistance, cancer stem cell development and tumour autophagy, contributing to the relapse of OC patients [39, 77-79, 95] (Table 2). In other types of cancer, ALKBH5 and FTO also participate in many pathological and biological activities, referring to proliferation, apoptosis, migration, invasion and metastasis [93, 94]. To date, a key set of studies have shown controversial roles of ALKBH5 in OC, which have also been observed in other cancer studies. We surmise that downstream signalling cascades are responsible for this apparent paradox, which will be discussed in the next section. Researchers have also found single-nucleotide polymorphisms (SNPs) in FTO and $A L K B H 5$, which may have important roles in breast cancer, melanoma, pancreatic cancer and endometrial cancer [96-99]. The state of SNPs of FTO and $A L K B H 5$ in OC has rarely been observed but may be of prognostic value and deserves further exploration.

Platinum-based chemotherapy is currently the first-line regimen for OC. PARPis have been licenced for treatment of patients with $B R C A$ mutations due to their substantial clinical benefits. Drug resistance to platinum and PARPis inevitably emerges, remaining a clinical hurdle because of unraveled underlying mechanisms. Nie et al. showed that ALKBH5 mRNA and protein expression upregulated in platinum-resistant EOC cells or samples, demonstrated by qRT-PCR, WB and IHC assays [39]. Moreover, CCK-8 and EdU assays indicated that ALKBH5 boosted proliferation and hindered apoptosis in A2780 and HO8910 cells (two EOC cell lines), which were confirmed in vivo through animal studies using xenograft mouse models [39]. Then, transcriptional analysis verified the presence of a homeobox A10 (HOXA10)-ALKBH5 loop involved in chemoresistance in EOC cells, which was confirmed by correlation analysis between ALKBH5 and HOXA10 expressions based on 483 EOCs in TCGA database
[39]. Further RIP-Seq, RNA-seq, RIP-qPCR and relative luciferase assays identified $J A K 2$ as an m6A demethylation substrate of ALKBH5, which was supported by data that blockade of either ALKBH5 or HOXA10 can rescue the activation of JAK2/STAT3 pathway [39]. This work underpins the importance of ALKBH5 in chemoresistance in $\mathrm{OC}$, which may be applied to $\mathrm{OC}$ prediction and development of new treatment strategies. Through RNAseq data, Fukumoto and the group found the whole levels of m6A-modified mRNA were similar between PARPiresistant and parental PEO1 cells [78]. By analysing differentially modified genes between PARPi-resistant and parental PEO1 cells, Fukumoto et al. identified frizzled class receptor 10 (FZD10) as the top gene with increased m6A modification due to downregulation of FTO and ALKBH5, which was demonstrated by MeRIP-qPCR, qRT-PCR and WB assays in PEO1 cells [78]. Further TCF/LEF dual luciferase reporter assays and dual HR and NHEJ reporter assays uncovered the downstream signalling pathway $\mathrm{Wnt} / \beta$-catenin, which was further validated by the synergistic effects on PARPi-resistant tumours in vivo, revealing that combining Wnt inhibitors overcomes PARPi resistance in BRCA-mutant OC [78]. This study casts further light upon the importance of FTO and ALKBH5 in resistance to PARPis. However, specific roles of FTO and ALKBH5 may be dependent on the type of drugs used and the mutation burden of $\mathrm{OC}$, which deserves further research.

The cancer stem cell (CSC) is a subgroup of cancer cells, featured by self-renewal and regeneration. Recent works have highlighted the important roles of CSCs in OC carcinogenesis, metastasis and resistance [100-102]. By comparing normal fallopian tube epithelium (FTE), OC tissues and normal ovary tissues, Huang and the group revealed that the mRNA level of FTO was significantly lower in ovarian tumours, which was further confirmed by analyses of TCGA Affymetrix Exon-array data (569 high-grade serous and 8 control FTE specimens) [77].

Table 2 The functions of m6A demethylases in OC

\begin{tabular}{|c|c|c|c|c|c|}
\hline Eraser & Expression & Function & Mechanism & Model & Ref. \\
\hline ALKBH5 & Up & Promotion & $\begin{array}{l}\text { ALKBH5-HOXA10 loop-mediates ALKBH5 expression, leading to JAK2 m6A demethyla- } \\
\text { tion and stabilization, thus OC resistance to cisplatin }\end{array}$ & In vitro; in vivo & [39] \\
\hline ALKBH5 & Up & Promotion & $\begin{array}{l}\text { In OC cells co-cultured with M2 macrophages, TLR4-NF-KB-mediated ALKBH5 upregula- } \\
\text { tion causes NANOG mRNA demethylation and stabilization }\end{array}$ & In vitro; in vivo & [79] \\
\hline ALKBH5/FTO & Down & Inhibition & $\begin{array}{l}\text { ALKBH5 and FTO downregulation elevates m6A-mediated stability of FZD10 mRNA and } \\
\text { causes PARPis resistance by activating Wnt/ } \beta \text {-catenin signalling in BRCA-mutated OC } \\
\text { cells }\end{array}$ & In vitro & {$[78]$} \\
\hline FTO & Down & Inhibition & $\begin{array}{l}\text { FTO suppression increases m6A methylation to stabilize PDE1C and PDE4B mRNA, thus } \\
\text { hindering CSC-related CAMP signalling }\end{array}$ & In vitro; in vivo & {$[77]$} \\
\hline FTO & Unknown & Unknown & $\begin{array}{l}\text { CircRAB1 1FIP1 promotes autophagy through FTO-mediated demethylation of ATG5 and } \\
\text { ATG7 }\end{array}$ & In vitro & {$[95]$} \\
\hline
\end{tabular}


Moreover, FTO levels were also significantly lower in the $\mathrm{ALDH}^{+}$cell population (a stem cell population) derived from OVCAR5 and COV362 cells [77]. Loss- and gainof-function experiments established an inhibitory role of FTO in self-renewal ability, colony formation ability, spheroid formation ability in vitro, and also the tumour initiation capacity in vivo [77]. Further integrative RNA sequencing and m6A mapping analyses revealed that cAMP signalling may be essential in hampering the stemness mediated by FTO blockade in OC [77]. In another study, through qRT-PCR analysis, Jiang et al. found upregulated $A L K B H 5$ in ovarian cancerous tissues than in normal tissues and downregulated $A L K B H 5$ in OC cell lines than in normal cells [79]. Further CCK-8 and flow cytometry assays showed protumour effects of ALKBH5 in ovarian cancer cells [79]. However, when cocultured with the M1 macrophage or the M2 macrophage, OC cells were divergently affected by ALKBH5 [79]. Specifically, the protumour abilities of OC cells were enhanced by coculture with M2 macrophages and inhibited by coculture with M1 macrophages. Collectively, Jiang et al. showed that OC cells upregulated ALKBH5 in inflammatory microenvironment to induce demethylation of Nanog homeobox (NANOG), which promoted the stemness and carcinogenesis of tumour cells [79]. The functions of demethylases are contradictory, which may be attributed to the complicated tumour microenvironment. Huang et al. observed that FTO downregulation was associated with cultures enriched with CSCs. Jiang et al. found that the effects of ALKBH5 on OC cells are dependent on a specific inflammatory microenvironment. These findings have advanced spirited debates on the effects of m6A regulators on interactions between tumours and their microenvironments. Continued work is important to clarify the effects and to benefit future m6A targeting strategies.

As a catabolic process, autophagy recycles cellular metabolites, macromolecules or organelles via lysosomes to maintain cellular homoeostasis [103-106]. A very large body of literature has established that autophagy is an important player during cancer development referring to proliferation, metastasis and resistance [107-110]. By using Torin 1-induced autophagy and sequencing in SK-OV-3 cells, Zhang et al. discovered that circRAB11FIP1 can promote autophagy, demonstrated by autophagy flux models (3-methyladenine, bafilomycin and Earle's Balanced Salt Solution) and autophagy readouts (the distribution of mRFP-GFP-LC3) in SK-OV-3 and A2780 cells [95]. Further RIP assays in SKOV3 cells revealed that circRAB11FIP1 could bind FTO mRNA through $14 \mathrm{bp}$, indicated by the greater enrichment of FTO mRNA in the circRAB11FIP1 WT group in SK-OV-3 than in the 14-bp mutant group [95].
This interaction subsequently enhanced the expression of FTO in A2780 and SK-OV-3, demonstrated by manipulations of circRAB11FIP1 expression in a qRT-PCR assay [95]. Interestingly, by employing multiple autophagy flux models and autophagy readouts, Wang et al. discovered that FTO promoted autophagy in 3T3-L1 cells. By conducting LC-MS/MS quantification, RIP-qPCR, WB, MeRIP-qPCR and firefly luciferase activity assays, the authors also found that FTO directly erased the m6A modification in mRNAs of Atg5 and Atg7 in 3T3-L1 cells, which was partially verified by Zhang et al. in human OC cells $[95,111]$. Moreover, in a generated fto-AKO model, deletion of Fto markedly inhibited autophagy, indicated by an attenuated LC3-II:I ratio, elevated Sqstm1 levels, and reduced Atg5 and Atg7 protein and gene expression $[95,111]$. These two studies highlight the vital role of FTO in autophagy. Considering that the functions of autophagy are highly context dependent, the specific role of FTO in cancer remains contradictory, despite the suppression of FTO in PARPi-resistant OC cells and CSC OC cells $[77,78,103]$.

\section{m6A methylation 'readers'}

In OC studies, m6A modification research mainly focuses on YTH domain family members (YTHDF1 and YTHDF1) and insulin-like growth factor 2 mRNAbinding proteins (IGF2BP1) [40, 80-82, 112]. Through functional and experimental analyses of ovarian cancer, recent studies underscore the protumour role of m6A 'readers' in vitro and in vivo, with regard to tumour growth, invasion and CSC phenotype [40, 80-82, 112] (Table 3). More recent work suggests that IGF2BP1, YTHDF1 and YTHDF1 mainly function as oncogenes in multiple cancer types, including colorectal cancer, hepatocellular carcinoma (HCC), gastric cancer, lung cancer, AML, pancreatic cancer, OC, bladder cancer, prostate cancer and melanoma $[73,113]$. However, in a few cases, IGF2BP1, YTHDF1 and YTHDF1 exert suppressive functions. IGF2BP1 hampers tumour cell growth and invasion in breast cancer [113]. YTHDF1 blocks the migration and growth of melanoma [73, 114]. YTHDF2 hinders the progression of melanoma as well as gastric cancer $[73,115$, 116]. These key sets of observations reinforce the context-dependent roles of $\mathrm{m} 6 \mathrm{~A}$ readers in multiple cancer types. This phenomenon is partly attributed to divergent functions of m6A targets, which can also be verified in $\mathrm{OC}$, as discussed below.

m6A methylation reading proteins can selectively bind modified products of $\mathrm{m} 6 \mathrm{~A}$ to affect the metabolism of mRNA. IGF2BP1 and YTHDF1 are implicated in $\mathrm{OC}$ progression by augmenting the translation of target mRNA [40, 81, 82]. A case in point is the previous discovery that IGF2BP1-serum response factor $(S R F)$ 
Table 3 The functions of m6A RNA binding proteins in OC

\begin{tabular}{|c|c|c|c|c|c|}
\hline Reader & Expression & Function & Mechanism & Model & Ref. \\
\hline IGF2BP1 & Unknown & Promotion & $\begin{array}{l}\text { In a 3'UTR- and m6A-dependent manner, IGF2BP1 promotes SRF expression to augment } \\
\text { SRF-dependent transcription }\end{array}$ & In vitro & {$[81]$} \\
\hline YTHDF1 & Unknown & Promotion & $\begin{array}{l}\text { YTHDF1 interacts with m6A-modified TRIM29 to promote TRIM29 expression, contributing } \\
\text { to CSC features in cisplatin-resistant OC cells }\end{array}$ & In vitro & [82] \\
\hline YTHDF1 & Up & Promotion & $\begin{array}{l}\text { In an m6A-dependent manner, YTHDF1 binds to m6A-modified EIF3C mRNA and aug- } \\
\text { ments EIF3C translation }\end{array}$ & In vitro; in vivo & {$[40]$} \\
\hline YTHDF2 & Up & Promotion & $\begin{array}{l}\text { YTHDF2, degraded by FBW7, can recognize m6A-modifed BMF mRNA and accelerate decay } \\
\text { of the latter }\end{array}$ & In vitro; in vivo & {$[80]$} \\
\hline YTHDF2 & Up & Promotion & $\begin{array}{l}\text { YTHDF2, repressed by miR-145, promotes OC progression by decreasing global mRNA m6A } \\
\text { levels }\end{array}$ & In vitro & [112] \\
\hline
\end{tabular}

mRNA association upregulates SRF expression, followed by its activation of oncogenic transcriptional function in HCC (Huh-7 cell line) and OC cells (ES-2 cell line) [81]. In this work, to identify effector networks of oncogenic IGF2BP1, the author employed RNA-seq after IGF2BP1 knockdown to monitor mRNA abundance and crosslinking immunoprecipitation high-throughput sequencing (CLIP-seq) studies to assess binding sites in Huh-7 and ES-2 cell lines [81]. Further gene set enrichment analysis predicted SRF mRNA as a target of IGF2BP1 in both Huh-7 and ES-2 cell lines, which was subsequently validated by IGF2BP1 knockout using CRISPR/Cas9 technology both in vitro and in vivo [81]. This regulation process was presumably m6A-dependent, as shown by the decrease in IGF2BP1-SRF mRNA association upon METTL3/14 depletion in cells [81]. Finally, the IGF2BP1SRF network promoted cell growth and invasion, demonstrated by a spheroid assay in cells, which was then verified by Kaplan-Meier Plot (Kmplot) analysis [81]. This work indicates that IGF2BP1 exerts an oncogenic role by targeting $S R F$ in OC. Similar to this discovery, Hao et al. reported that YTHDF1 was recruited to m6A-modified tripartite motif containing 29 (TRIM29) to upregulate TRIM29 expression, which empowered cisplatin-resistant OC cells (A2780/DDP and SK-OV-3/ DDP) with a CSC phenotype [82]. Using SILAC-labelled biotin pulldown assays followed by quantitative mass spectrometry, Hao et al. identified YTHDF1 as a TRIM29 mRNA-binding protein, which was further established through a RIP assay, luciferase assay, WB and RIP-qPCR assay in A2780/DDP and SK-OV-3/DDP cells [82]. Next, functional analyses (including colony formation, Transwell analysis and a spheroid formation assay) indicated that YTHDF1 knockdown significantly suppressed the CSC features of A2780/DDP and SK-OV-3/DDP cells but showed no effects on the parental cells, which was consistent with the rescue environment induced by TRIM29 overexpression [82]. This study suggests that YTHDF1 behaves as an oncogene in tumours by targeting TRIM2 9 in cisplatin-resistant OC cells. In another similar study, by analysing several cohorts of OC patients in TCGA pan-cancer database and GEO datasets (GSE66957 and GSE54388), Liu et al. discovered that YTHDF1 was elevated in various cancer types, including ovarian cancer, which was further established by qRT-PCR analysis of human fresh frozen ovarian tissues $(\mathrm{n}=35$ for cancerous; $\mathrm{n}=12$ for normal) and by IHC data from an OC tissue microarray $(\mathrm{n}=134)$ [40]. Next, Kmplot survival and FIGO stage analyses revealed that the expression of YTHDF1 significantly correlated with the prognosis of OC patients [40]. Functional analyses, including CCK-8, EdU staining, colony formation and Transwell assays, showed that YTHDF1 knockdown suppressed cell growth and migration in A2780 and SK-OV-3 cells in vitro, which was validated in vivo in a xenograft mouse model and an orthotopic mouse model [40]. Multiomics analyses, including RNA-seq, RIP-seq, eCLIP-seq and CLIP-qPCR, identified EIF3C as a YTHDF1-binding transcript that regulates RNA translation, which was consistent with the unchanged RNA abundance of YTHDF1 targets in multiomics analyses and was also further confirmed by the mutation of YTHDF1 and rescue experiments [40]. This study revealed that the oncogene EIF3C is a target mRNA for the m6A reader YTHDF1 in OC. In a word, these studies indicate that IGF2BP1 and YTHDF1 exert their oncogenic roles through their targets in OC, which provides opportunities to develop new diagnostic markers and new strategies to target IGF2BP1 and YTHDF1 in OC.

Early and more recent literature underlines the critical role of m6A regulators in OC. Exploration of downstream signalling cascades of the m6A regulator may answer the question that how $\mathrm{m} 6 \mathrm{~A}$ regulators function during $\mathrm{OC}$ progression [40,81, 82]. There is another question: why are m6A regulators aberrantly expressed in OC? Explorations of upstream m6A regulators may elucidate this question. Recently, Xu et al. identified YTHDF2 as a novel substrate for E3-ubiquitin ligase (F-box and 
WD repeat domain containing 7) FBW7 in OC, the latter of which is a tumour suppressor [80]. After degradation via the proteasome system, impaired YTHDF2 impinged on mRNA decay of the proapoptotic gene bcl2 modifying factor $(B M F)$ in vitro and in vivo [80]. In this study, by analysing data from TCGA RNA-Seq database ( $\mathrm{n}$ $=1207)$, Xu et al. displayed that YTHDF2 was elevated in the ovarian cancerous tissue and closely associated with poor prognosis of OC patients. This is in line with results from OC tissue microarrays $(\mathrm{n}=115)$ analyses via qRT-PCR, IHC and Kmplot survival analyses [80]. In addition, both in vitro and in vivo, blockade of YTHDF2 suppressed the growth of OC cells, as shown by CCK- 8 assays, colony formation assays and a mouse xenograft model [80]. Further mechanistic research identified FBW7 as the E3-ubiquitin ligase for YTHDF2 and identified $B M F$ mRNA as the m6A-modified substrate in OC [80]. Another clue can also be found by looking at an upstream factor of YTHDF2, namely, miR-145, which can hinder the abilities of OC cells to proliferate and migrate [112]. In this study, by analysing the ovarian cancerous tissue $(\mathrm{n}=31)$ and the normal ovarian tissue $(\mathrm{n}=14)$, $\mathrm{Li}$ et al. showed that increments of YTHDF2 protein in the ovarian cancerous tissue was closely related to clinical stages [112]. Moreover, functional analyses via CCK8, Transwell and Annexin V-FITC/propidium iodide staining analyses revealed that YTHDF2 conferred the abilities to proliferate and migrate on OC cells (SK-OV-3 and $3 \mathrm{AO}$ cells) [112]. Further TargetScan prediction, luciferase reporter, WB and qRT-PCR analyses showed that $Y T H D F 2$ may be a direct target of miR-145, whose protumour ability could be rescued by YTHDF2 overexpression in SK-OV-3 and 3AO cells [112]. This crosstalk also occurred in HCC, in which miR-145 can directly target $3^{\prime}$ UTR in YTHDF2 mRNA to block YTHDF2 expression [117]. These two studies indicate tumour-promoting effects of YTHDF2 and dissect the upstream signalling in $\mathrm{OC}$, redefining $\mathrm{m} 6 \mathrm{~A}$ modification as a signalling hub orchestrating several important pathways, such as the ubiquitin-proteasome system and miRNA.

\section{The double-edged sword role of m6A modification in OC}

Demethylase knockdown has the same effect as methylase knockdown on m6A modification, and vice versa, which has been confirmed in multiple rescue experiments. As proof of this fact, $\mathrm{m} 6 \mathrm{~A}$ in transcription factor EB (TFEB) mRNA is balanced by both ALKBH5 and METTL3 [118]. In fact, the same effects on m6A modification have opposite biological functions in lung cancer, breast cancer and AML [21, 119]. Generally, in lung cancer, FTO is elevated to promote proliferation and invasion. Specifically, FTO can erase the m6A in ubiquitin specific peptidase 7 (USP7) and myeloid zinc finger 1 (MZF1) mRNAs, resulting in USP7 and MZF1 overexpression, which function as oncogenes in lung cancer [120, 121]. However, Du et al. reported METTL3 rescued miR-338-5p-mediated growth and invasion of lung cancer cells [122]. Breast cancer also presents this paradox. Through METTL14 overexpression or ALKBH5 knockdown experiments, some researchers have found that increasing global m6A levels impede propagation of human breast cancer cells [123]. Other researchers have discovered that increased global m6A levels were present in breast cancer patients and that METTL3 knockdown enhances apoptosis by hindering $\mathrm{m} 6 \mathrm{~A}$ modification of the oncogene $\mathrm{Bcl}-2$ and its translation [124]. In AML, demethylases (FTO and ALKBH5) possess the ability to inhibit disease progression in specific situations, so do methyltransferases (METTL3, WTAP and METTL14) [125-129]. These findings reveal the functional inconsistency of m6A modification in different cancers, including lung cancer, breast cancer and AML.

This paradox is also observed during OC progression. Almost all experimental data show METTL3 contributes to $\mathrm{OC}$ progression by participating in multiple signalling pathways, including the $A K T, E I F 3 C, A X L, C S F-1$ and FZD10 pathways, predicting poor prognosis for OC patients [35-37, 76, 83]. Consistent with these discoveries regarding m6A levels, ALKBH5 and FTO were impaired to elevate m6A modification of targeted genes and accelerate tumour progression [77, 78]. In $B R C A$ -mutant OC cells, ALKBH5 and FTO downregulation led to PARPi resistance through stabilization of FZD10 mRNA [78]. In OC stem cells, FTO enhanced cAMP signalling to impede the stemness features of $\mathrm{OC}$ via reducing the stability of phosphodiesterase $1 C(P D E 1 C)$ and phosphodiesterase 4B (PDE4B) mRNA [77]. Contradictory discoveries have revealed that ALKBH5 overexpression endows $\mathrm{OC}$ cells with the ability to resist cisplatin and maintain stemness via separate m6A demethylation of JAK2 and NANOG [39, 79]. Altogether these studies further reflect the contradictory roles of m6A modification in OC.

These paradoxes have raised a new question as to why the same m6A modification has opposite biological functions in $\mathrm{OC}$ and other cancer types. Considering that $\mathrm{m} 6 \mathrm{~A}$ readers fulfil the functions of writers and erasers, researchers have surmised that $\mathrm{m} 6 \mathrm{~A}$ readers may point to opposite functions caused by the same m6A modification, which is supported by the opposite biological functions of the same eraser ALKBH5 in OC. We speculate that downstream signalling cascades also contribute to the 'double-edged sword' role of m6A modification in OC. One of the reasons stems from the different targets (JAK2 and FZD10) of the 
demethylase ALKBH5 reported respectively by Nie et al. and Fukumoto et al. in OC [39, 78]. Although the two studies both focused on drug resistance, the different expressions of ALKBH5 were found by Nie et al. in cisplatin-resistant EOC cells and by Fukumoto et al. in BRCA2-mutated PARPi resistant OC cells, which displayed distinct targets of ALKBH5. Another salient example lies in glioblastoma; METTL3/14 knockdown can promote tumorigenesis for glioblastoma stem cell (GSC) by decreasing m6A modification in the mRNA of the targeted oncogene ADAM metallopeptidase domain 19 (ADAM19) [130]. Paradoxically, it has been reported that METTL3 is essential to GSC maintenance and radioresistance through upregulation of the targeted mRNA of the oncogene SRY-box transcription factor 2 (SOX2) [131]. Although the two studies both focused on GSC phenotype, Cui et al. and Visvanathan et al. both used primary GSCs cultured as 3D tumourspheres but from different patients, which directed METTL3 to different targets and opposite roles in glioblastoma. These two paired studies demonstrate that apparent paradoxical roles of m6A signaling may finally converge on the targets of m6A modification in OC. Another issue that sheds light on the 'double-edged sword' role of m6A during OC progression refers to context-dependent functions of downstream signalling, such as autophagy. Autophagy is a catabolic process in which the endoplasmic reticulum forms a double membrane, creating a phagophore to engulf cellular cargo that subsequently fuses with a lysosome to produce an autolysosome, finally resulting in degradation of the cargo [107]. Several researchers have reviewed the pleiotropic functions of autophagy and identified autophagy as a 'Janus-faced' player in cancer development [132], which may partially dictate paradoxical roles of m6A modification during $\mathrm{OC}$ progression.

In conclusion, as shown by present evidence, m6A modification exerts opposite functions during OC progression, which relies on contradictory effects from two aspects: $\mathrm{m} 6 \mathrm{~A}$ regulators and their downstream signalling cascades.

\section{Opportunities for application of m6A modification in OC}

Among myriads of distinct epigenetic modifications, m6A modification is the most prevalent and has the potential to solve the clinical problems related to $\mathrm{OC}$ treatment. One application of m6A modification is early detection and prognosis prediction in $\mathrm{OC}$ patients. Another application of m6A modification is to develop m6A-targeted therapeutics for OC patients.
Early detection and prognosis prediction in $O C$ patients Multiomics studies and bioinformatics analyses of OC have explored the landscape features and prognostic values of m6A regulators. By integrating multiple databases, including GEO, TIMER, ROC Plotter and Kmplot, a study by Wang indicated that HNRNPC could predict relapse for patients with paclitaxel treatment; decreased $Y T H D C 1$ and increased $R B M 15$ expression could predict metastasis; and RBM15B, ZC3H13, YTHDF1 and $I G F 2 B P 1$ were closely associated with the immunological characteristics of OC patients [133]. Fan et al. underscored the important role of a three-gene signature (IGF2BP1, VIRMA and ZC3H13) for prognosis prediction [134]. Another four-gene signature reported by Wei et al. included HNRNPA2B1, which played an antitumour role [135]. Despite its good performance for prognosis prediction in both the training set and test set, the same author previously reported that HNRNPA2B1 played a protumour role in OC [136]. This paradox is explained not only by the dual role of m6A regulators but also by the inconsistency between protein and mRNA levels, which was also confirmed by Fan et al. [134]. In the study reported by Fan et al., compared with the normal tissue, the mRNA as well as protein expression level of ZC3H13 upregulated in the cancerous tissue, whereas the protein levels of IGF2BP1 and VIRMA differed from their mRNA levels [134]. In a word, there is an urgent need to conduct much more experiments to apply m6A modification to early detection or prognosis prediction.

Taking the current experimental evidence into consideration, METTL3 may be a proper marker for early detection and prognosis prediction in $\mathrm{OC}$ patients. However, there is limited clinical evidence showing correlations between different histotypes of $\mathrm{OC}$ tissues and METTL3 expression. How METTL3 expression compares with CA-125 concentration, which is currently the tumour marker widely used for early detection and prognosis prediction for $\mathrm{OC}$ patients, is still unknown.

\section{Possible strategies to target m6A modification in OC}

Despite the clinical success of inhibitors of DNA epigenetics, including methyltransferases, to date, there are no approved drugs to target m6A RNA modification.

Early studies on m6A regulator-based targeting strategies mainly focused on demethylases. ALKBH5 and FTO exert their function via interacting with their cofactors and substrates, which are blocked by most reported inhibitors of FTO or ALKBH5 (such as rhein, CHTB, N-CDPCB, FB23-2, CS1/2, meclofenamic acid and entacapone) [137]. By screening studies of approved drugs, Huang et al. and Peng et al. separately identified meclofenamic acid (a nonsteroidal anti-inflammatory 
drug) and entacapone (combines with levodopa to treat Parkinson) as originally discovered inhibitors of FTO [138, 139]. FB23-2, a derivative of meclofenamic acid, was further demonstrated to suppress the propagation of human AML in vitro as well as in vivo [140]. Peng et al. revealed that one target of FTO in gluconeogenesis and thermogenesis was the transcription factor forkhead box protein O1 (FOXO1) mRNA, which participated in BCR-ABL1-independent imatinib relapse [141]. Meclofenamic acid and entacapone separately entered clinical trials for recurrent metastasis (NCT02429570) and gastrointestinal stromal tumours combined with imatinib (NCT04006769), highlighting the possibility of targeting m6A regulators for advanced or relapsed cancer [137]. Radicicol inhibits FTO in a dose-dependent manner (IC50 $=16.04 \mu \mathrm{M}$ in enzymatic assays), which could enhance TRAIL-induced apoptosis in OC cells [142, 143]. Although these results hint at the possibility that radicicol may have antitumour effects in OC cells under specific conditions, inhibitors of m6A methyltransferases and demethylases have not been studied in $\mathrm{OC}$ and deserve in-depth research.

There are also several studies contributing to targeting of METTL3, and inhibitors of other writers are extremely rare. Through virtual screenings, Selberg et al. first identified four compounds that activated the METTL314-WTAP complex and led to the greatest increase in m6A level (21.4 $\pm 12.9 \%)$ in cellular assays [144]. Using a cofactor mimicking approach, Bedi et al. also discovered 7 potential inhibitors of METTL3 in silico [145]. Compound 2 was considered to be the most potential, with IC50 $=8.7 \mu \mathrm{M}$ in enzymatic assays [145]. No cellular data were obtained in Bedi's work. To date, there are two different selective small molecule inhibitors of METTL3, namely, STM2457 and UZH1a, which were independently reported by Yankova et al. and Moroz-Omori et al. [146, 147]. When binding with METTL3, the two inhibitors both occupy the SAM-binding site and reorganize Lys 513 of METTL3, which partly contributes to their selectivity. Consistently, SAM and sinefungin, pan inhibitors of methyltransferases, do not possess a specific binding mode [148]. Although the activity of STM2457 and UZH1a in OC is still unknown, both STM2457 and UZH1a could dampen the activity of METTL3 in the AML cell line MOLM-13 [146, 147]. Moreover, the propagation of several AML cell lines from human or mouse was blocked by STM2457 [146]. The efficacy of STM2457 was evaluated in a PDX model as well as a primary murine model, both of which carried different cancer drivers in vivo [146]. STM2457 displayed promising antileukaemic effects due to impaired AML propagation, diminishment of stem cells and improved survival with STM2457 treatment. Similar to AML, recent studies have also identified METTL3 as an oncogene in OC, providing important insights for exploration of METTL3 inhibitors in $\mathrm{OC}$, which deserves further investigations.

To develop m6A regulator-based inhibitors for OC, researchers may consider the important question of which targeting technology is applicable. Considering that m6A-based RNA methyltransferases and demethylases may have catalytic-independent roles in OC, especially METTL3, proteolysis targeting chimaera (PROTAC) may be a promising technology to degrade this type of m6A regulator, which is consistent with the advice of Zeng et al. [50]. The first oral PROTAC (NCT03888612 for ARV-110, NCT04072952 for ARV471) entered phase I clinical trials for prostate and breast cancer treatment separately and displayed promising data, showing good safety and efficacy [149]. The antitumour effects of PROTACs in OC have been established to target PI3K in OVCAR8 cells in vitro as well as in vivo, highlighting the advantages of PROTACs [150]. These data open new possibilities for targeting $\mathrm{m} 6 \mathrm{~A}$ regulators using the PROTAC technology.

m6A modification ultimately fine tunes the destination of mRNA, shedding light upon targeting mRNA processing as an emerging anticancer strategy, which may ignore the catalytic-independent role of methylases in OC. Alternative splicing is a key step in mRNA processing and participates in tumour invasion, proliferation, metabolism and drug resistance [151-155]. Specifically, in OC, a splice variant of BRCA1 encodes BRCA1- $\triangle 11 \mathrm{q}$, resulting in resistance to PARPis compared with full-length BRCA1 in PDX models, which could be solved by targeting splicing [156]. As previously summarized, m6A-modified JAK2, FZD10 and TRIM29 all contribute to resistance to PARPis and cisplatin. Considering $\mathrm{m} 6 \mathrm{~A}$ as a signalling to modulate splicing [41, $42,157]$, these observations are reminiscent of the question of whether splicing abnormalities exist for these m6A-modified mRNAs or other mRNAs (in addition to BRCA1) in OC $[156,158]$. Although several splicing modulators have entered phase I clinical trials for cancer treatments, as reviewed by Desterro (e.g., NCT02841540 and NCT03614728) [159], this question is still important to employ splicing modulating as a strategy to target m6A-modified mRNA in OC. Another strategy targeting mRNA processing is therapeutic oligonucleotides with chemical modifications that possess the ability to inhibit gene expression [160]. To date, four therapeutic oligonucleotides have entered the clinic, mainly approved for non-tumour diseases. Many antitumour oligonucleotides have also been developed and evaluated in clinical trials, including Apatorsen (NCT01454089), AZD9150 (NCT02549651 and NCT01563302), AZD5312 (NCT02144051), MIR155 (NCT02580552), Custirsen 
(NCT01578655 and NCT01630733) and EZN-4176 (NCT01337518). However, the study of therapeutic oligonucleotides in OC is still deficient. There are also obstacles to applying this technology to OC. For example, which m6A-modified mRNA should be chosen? How can m6A-modified mRNA be specifically targeted. By solving these problems, therapeutic oligonucleotides are likely to become a hot research topic for development of RNAbased therapeutics to treat OC.

\section{Conclusion}

In summary, aberrant m6A levels in $\mathrm{OC}$, modulated by $\mathrm{m} 6 \mathrm{~A}$ regulators, participate in $\mathrm{OC}$ progression and predict relapse for OC patients. Considering the insights recently acquired, we can conclude several points: below. First, METTL3 is essential to the methyltransferase complex and acts as a protumour role during OC progression through catalytic-dependent or catalytic-independent pathways. The functions and modes of other methyltransferase complexes in OC remain an enigma. Second, m6A modification regulators have pleiotropic contextdependent functions in $\mathrm{OC}$, indicating contradictory roles of $\mathrm{m} 6 \mathrm{~A}$ regulators. The mechanisms underlying this apparent paradox need to be further explored and may involve upstream stimuli, downstream signalling cascades, cancer cells and the tumour microenvironment. Last, similar to protein posttranslational modifications, crosstalk exists among m6A modifications and other RNA modification forms, which may partly contribute to the complexity of OC progression.

Collectively, this review not only updates our knowledge of m6A modification in OC, highlighting the important roles of $\mathrm{m} 6 \mathrm{~A}$ regulators and the underlying mechanisms, but also provides a rationale for development of new diagnostic markers and therapeutic strategies based on m6A modifications and regulators in OC.

\footnotetext{
Abbreviations

2OG: 2-Oxoglutarate; ADAM19: ADAM metallopeptidase domain 19; ALKBH5: AlkB homolog 5, RNA demethylase; AML: Acute myeloid leukaemia; AXL: AXL receptor tyrosine kinase; BMF: BCI2 modifying factor; CCR4-NOT: Carbon catabolite repression 4-negative on TATA-less deadenylase complex; CEBPZ: CCAAT enhancer binding protein zeta; CLIP-seq: Cross-linking immunoprecipitation high-throughput sequencing; CSCs: Cancer stem cells; PDE1C: Phosphodiesterase 1C; EIF3C: Eukaryotic translation initiation factor 3 subunit C; EMT: Epithelial to mesenchymal transition; FBW7: F-box and WD repeat domain containing 7; FMR1: Fragile X mental retardation 1; FOXO3: Forkhead box O3; UTR: Untranslated terminal region; FTE: Fallopian tube epithelium; FTO: Fat mass and obesity-associated protein; FZD10: Frizzled class receptor 10; HNRNP: Heterogeneous nuclear ribonucleoprotein binding protein; HOXA10: Homeobox A10; HSPH1: Heat shock protein family H (Hsp110) member 1; ICC: Intrahepatic cholangiocarcinoma; IGF2BP2: Insulin like growth factor 2 mRNA binding protein 2; IHC: Immunohistochemistry; JAK2: Janus kinase 2; Kmplot: Kaplan-Meier Plot; KIAA1429/VIRMA: Vir like m6A methyltransferase associated; LRPPRC: Leucine rich pentatricopeptide repeat containing; m6A: N6-methyladenosine; m6Am: N6,2'-O-dimethyladenosine; METTL3: Methyltransferase-like 3; METTL14: Methyltransferase-like 14; METTL16:
}

Methyltransferase-like 16; mTOR: Mammalian target of the rapamycin; MZF1: Myeloid zinc finger 1; NANOG: Nanog homeobox; OC: Ovarian cancer; PARPi: Inhibitors of poly (ADP-ribose) polymerase; PD-L1: Programmed cell death-1 ligand-1; PDE4B: Phosphodiesterase 4B; SRF: Serum response factor; PROTAC : Proteolysis targeting chimaera; PI3K: Phosphoinositide 3-kinase; AKT: AKT serine/threonine kinase 1; PTEN: Phosphatase and tensin homolog; qRT-PCR: Real-time quantitative polymerase chain reaction; RBM15: RNA binding motif protein 15; RCC: Renal cell carcinoma; RHPN1-AS1: RHPN1 antisense RNA 1 (head to head); RIP: RNA immunoprecipitation; SAM: S-adenosylmethionine; SETDB1: SET domain bifurcated histone lysine methyltransferase 1; SNPs: Single-nucleotide polymorphisms; SOCSs: Suppressor of cytokine signalling family genes; SOX2: SRY-box transcription factor 2; STAT3: Signal transducer and activator of transcription 3; YTHDC1:YTH domain containing 1; TFEB: Transcription factor EB; USP7: Ubiquitin specific peptidase 7; TRIM29: Tripartite motif containing 29; WB: Western blot; WTAP: Wilms tumor 1-associated protein; YTHDF1:YTH N6-methyladenosine RNA binding protein 1; ZC3H13: Zinc finger CCCH domain-containing protein 13.

\section{Acknowledgements \\ Not applicable.}

\section{Authors' contributions}

Conceiving and designing the manuscript: $L C, B H$ and $W Z$; literature retrieval: QG and YF; writing of the manuscript: $L C, X X, X L, Q G$ and $Y F$; drawing: $L C$ and $X \mathrm{~L}$; reviewed and revision: $\mathrm{BH}$ and $\mathrm{WZ}$; final approval: $\mathrm{LC}, \mathrm{XX}, \mathrm{XL}, \mathrm{QG}, \mathrm{YF}, \mathrm{BH}$ and WZ. All authors read and approved the final manuscript.

\section{Funding}

This work was supported by the Science and Technology Research Project of Henan Province (No. 212102311032); the Henan Provincial Medical Science and Technology Project (No. LHGJ20210178).

Availability of data and materials

Not applicable.

\section{Declarations}

Ethics approval and consent to participate

Not applicable.

Consent for publication

Not applicable.

\section{Competing interests}

The authors declare no competing interests.

\section{Author details}

${ }^{1}$ Department of Pharmacy, Affiliated Tumour Hospital of Zhengzhou University, Henan Cancer Hospital, 127\# Dongming Rd, Zhengzhou 450008, Henan, China. ${ }^{2}$ Department of Clinical Pharmacy, Zhengzhou Central Hospital Affiliated To Zhengzhou University, Zhengzhou, China.

Received: 29 September 2021 Accepted: 25 November 2021

Published online: 11 December 2021

\footnotetext{
References

1. Poveda A, Ray-Coquard I, Romero I, Lopez-Guerrero JA, Colombo N. Emerging treatment strategies in recurrent platinum-sensitive ovarian cancer: focus on trabectedin. Cancer Treat Rev. 2014;40(3):366-75.

2. Fang J, Ding N, Guo X, Sun Y, Zhang Z, Xie B, et al. alphaPD-1-mesoCAR$T$ cells partially inhibit the growth of advanced/refractory ovarian cancer in a patient along with daily apatinib. J Immunother Cancer. 2021. https://doi.org/10.1136/jitc-2020-001162.

3. Armstrong DK, Alvarez RD, Bakkum-Gamez JN, Barroilhet L, Behbakht K, Berchuck A, et al. Ovarian cancer, version 2.2020, NCCN clinical practice guidelines in oncology. J Natl Compr Canc Netw. 2021;19(2):191-226.

4. Kujawa KA, Lisowska KM. Ovarian cancer — from biology to clinic. Postepy Hig Med Dosw. 2015;69:1275-90.
} 
5. Furuya M. Ovarian cancer stroma: pathophysiology and the roles in cancer development. Cancers. 2012;4(3):701-24.

6. Kaldawy A, Segev Y, Lavie O, Auslender R, Sopik V, Narod SA. Low-grade serous ovarian cancer: a review. Gynecol Oncol. 2016;143(2):433-8.

7. Haight P, Savage J, Bixel K. The poor prognosis of sarcomatoid carcinoma arising from low grade serous ovarian cancer: a case report and review of the literature. Gynecol Oncol Rep. 2021;36:100735.

8. Burki TK. CA-125 blood test in early detection of ovarian cancer. Lancet Oncol. 2015:16(6):e269.

9. Killock D. Gynaecological cancer: biomarker potential of CA-125 enhanced. Nat Rev Clin Oncol. 2015;12(8):437.

10. Kuroki L, Guntupalli SR. Treatment of epithelial ovarian cancer. BMJ. 2020;371:m3773.

11. Delgado Bolton RC, Aide N, Colletti PM, Ferrero A, Paez D, Skanjeti A, et al. EANM guideline on the role of 2-[(18)F]FDG PET/CT in diagnosis, staging, prognostic value, therapy assessment and restaging of ovarian cancer, endorsed by the American College of Nuclear Medicine (ACNM), the Society of Nuclear Medicine and Molecular Imaging (SNMMI) and the International Atomic Energy Agency (IAEA). Eur J Nucl Med Mol Imaging. 2021:48(10):3286-302

12. Feng W, Dean DC, Hornicek FJ, Shi H, Duan Z. Exosomes promote pre-metastatic niche formation in ovarian cancer. Mol Cancer. 2019;18(1):124.

13. Lorusso D, Ceni V, Daniele G, Salutari V, Pietragalla A, Muratore M, et al. Newly diagnosed ovarian cancer: which first-line treatment? Cancer Treat Rev. 2020;91:102111.

14. Moore K, Colombo N, Scambia G, Kim BG, Oaknin A, Friedlander M, et al. Maintenance olaparib in patients with newly diagnosed advanced ovarian cancer. N Engl J Med. 2018;379(26):2495-505.

15. Ray-Coquard I, Pautier P, Pignata S, Perol D, Gonzalez-Martin A, Berger $\mathrm{R}$, et al. Olaparib plus bevacizumab as first-line maintenance in ovarian cancer. N Engl J Med. 2019;381(25):2416-28.

16. Gonzalez-Martin A, Pothuri B, Vergote I, DePont CR, Graybill W, Mirza $M R$, et al. Niraparib in patients with newly diagnosed advanced ovarian cancer. N Engl J Med. 2019;381(25):2391-402.

17. Tsibulak I, Zeimet AG, Marth C. Hopes and failures in front-line ovarian cancer therapy. Crit Rev Oncol Hematol. 2019;143:14-9.

18. Khan MA, Vikramdeo KS, Sudan SK, Singh S, Wilhite A, Dasgupta S, et al. Platinum-resistant ovarian cancer: from drug resistance mechanisms to liquid biopsy-based biomarkers for disease management. Semin Cancer Biol. 2021. https://doi.org/10.1016/j.semcancer.2021.08.005.

19. Xie W, Sun H, Li X, Lin F, Wang Z, Wang X. Ovarian cancer: epigenetics, drug resistance, and progression. Cancer Cell Int. 2021;21(1):434.

20. Fu Y, Dominissini D, Rechavi G, He C. Gene expression regulation mediated through reversible m(6)A RNA methylation. Nat Rev Genet. 2014;15(5):293-306

21. Wang $S$, Chai P, Jia R, Jia R. Novel insights on m(6)A RNA methylation in tumorigenesis: a double-edged sword. Mol Cancer. 2018;17(1):101.

22. Hastings MH. m(6)A mRNA methylation: a new circadian pacesetter. Cell. 2013;155(4):740-1.

23. Kumari R, Ranjan P, Suleiman ZG, Goswami SK, Li J, Prasad R, et al. mRNA modifications in cardiovascular biology and disease: with a focus on m6A modification. Cardiovasc Res. 2021. https://doi.org/10.1093/cvr/ cvab160.

24. Huang W, Chen TQ, Fang K, Zeng ZC, Ye H, Chen YQ. N6-methyladenosine methyltransferases: functions, regulation, and clinical potential. J Hematol Oncol. 2021;14(1):117.

25. Liu Z, Chen X, Zhang P, Li F, Zhang L, Li X, et al. Transcriptome-wide dynamics of $m(6) A$ mRNA methylation during porcine spermatogenesis. Genom Proteom Bioinform. 2021. https://doi.org/10.1016/j.gpb. 2021.08.006.

26. Zhou J, Wan J, Gao X, Zhang X, Jaffrey SR, Qian SB. Dynamic m(6)A mRNA methylation directs translational control of heat shock response. Nature. 2015:526(7574):591-4

27. Ninomiya K, Iwakiri J, Aly MK, Sakaguchi Y, Adachi S, Natsume T, et al. m(6) A modification of HSATIII IncRNAs regulates temperature-dependent splicing. EMBO J. 2021;40(15):e107976.

28. Wang J. Trapping m6A proteins for splicing regulation. Nat Cell Biol. 2021;23(8):811.
29. Li HB, Tong J, Zhu S, Batista PJ, Duffy EE, Zhao J, et al. m(6)A mRNA methylation controls $T$ cell homeostasis by targeting the IL-7/STAT5/ SOCS pathways. Nature. 2017;548(7667):338-42.

30. Gui Y, Yuan S. Epigenetic regulations in mammalian spermatogenesis: RNA-m(6)A modification and beyond. Cell Mol Life Sci. 2021;78(11):4893-905.

31. Wang L, Song C, Wang N, Li S, Liu Q, Sun Z, et al. NADP modulates RNA m(6)A methylation and adipogenesis via enhancing FTO activity. Nat Chem Biol. 2020:16(12):1394-402.

32. Chokkalla AK, Mehta SL, Vemuganti R. Epitranscriptomic regulation by m(6)A RNA methylation in brain development and diseases. J Cereb Blood Flow Metab. 2020:40(12):2331-49.

33. Aguilo F, Zhang F, Sancho A, Fidalgo M, Di Cecilia S, Vashisht A, et al. Coordination of $\mathrm{m}(6) \mathrm{A}$ mRNA methylation and gene transcription by ZFP217 regulates pluripotency and reprogramming. Cell Stem Cell. 2015;17(6):689-704.

34. Barbieri I, Tzelepis K, Pandolfini L, Shi J, Millan-Zambrano G, Robson SC, et al. Promoter-bound METTL3 maintains myeloid leukaemia by m(6) A-dependent translation control. Nature. 2017;552(7683):126-31.

35. Wang J, Ding W, Xu Y, Tao E, Mo M, Xu W, et al. Long non-coding RNA RHPN1-AS1 promotes tumorigenesis and metastasis of ovarian cancer by acting as a ceRNA against miR-596 and upregulating LETM1. Aging. 2020;12(5):4558-72.

36. Bi X, LV X, Liu D, Guo H, Yao G, Wang L, et al. METTL3-mediated maturation of miR-126-5p promotes ovarian cancer progression via PTEN-mediated PI3K/Akt/mTOR pathway. Cancer Gene Ther. 2021:28(3-4):335-49.

37. Hua W, Zhao Y, Jin X, Yu D, He J, Xie D, et al. METTL3 promotes ovarian carcinoma growth and invasion through the regulation of $A X L$ translation and epithelial to mesenchymal transition. Gynecol Oncol. 2018:151(2):356-65.

38. Qiu X, Yang S, Wang S, Wu J, Zheng B, Wang K, et al. M(6)A demethylase ALKBH5 regulates PD-L1 expression and tumor immunoenvironment in intrahepatic cholangiocarcinoma. Cancer Res. 2021;81 (18):4778-93.

39. Nie S, Zhang L, Liu J, Wan Y, Jiang Y, Yang J, et al. ALKBH5-HOXA10 loopmediated JAK2 m6A demethylation and cisplatin resistance in epithelial ovarian cancer. J Exp Clin Cancer Res. 2021;40(1):284

40. Liu T, Wei Q, Jin J, Luo Q, Liu Y, Yang Y, et al. The m6A reader YTHDF1 promotes ovarian cancer progression via augmenting EIF3C translation. Nucleic Acids Res. 2020;48(7):3816-31.

41. Adhikari S, Xiao W, Zhao YL, Yang YG. m(6)A: signaling for mRNA splicing. RNA Biol. 2016:13(9):756-9.

42. Mendel M, Delaney K, Pandey RR, Chen KM, Wenda JM, Vagbo CB, et al. Splice site m(6)A methylation prevents binding of U2AF35 to inhibit RNA splicing. Cell. 2021;184(12):3125-3142 e3125.

43. Zhang C, Fu J, Zhou Y. A review in research progress concerning m6A methylation and immunoregulation. Front Immunol. 2019;10:922.

44. Wei CM, Gershowitz A, Moss B. Methylated nucleotides block 5 ' terminus of HeLa cell messenger RNA. Cell. 1975;4(4):379-86.

45. Zaccara S, Ries RJ, Jaffrey SR. Reading, writing and erasing mRNA methylation. Nat Rev Mol Cell Biol. 2019;20(10):608-24.

46. Wang P, Doxtader KA, Nam Y. Structural basis for cooperative function of METTL3 and METTL14 methyltransferases. Mol Cell. 2016;63(2):306-17.

47. Dominissini D, Moshitch-Moshkovitz S, Schwartz S, Salmon-Divon M, Ungar L, Osenberg $\mathrm{S}$, et al. Topology of the human and mouse m6A RNA methylomes revealed by m6A-seq. Nature. 2012;485(7397):201-6.

48. Meyer KD, Jaffrey SR. Rethinking $\mathrm{m}(6) \mathrm{A}$ readers, writers, and erasers. Annu Rev Cell Dev Biol. 2017:33:319-42.

49. Deng $X$, Su R, Weng H, Huang H, Li Z, Chen J. RNA N(6)-methyladenosine modification in cancers: current status and perspectives. Cell Res. 2018;28(5):507-17.

50. Zeng C, Huang W, Li Y, Weng H. Roles of METTL3 in cancer: mechanisms and therapeutic targeting. J Hematol Oncol. 2020;13(1):117.

51. Wang $X$, Feng J, Xue Y, Guan Z, Zhang D, Liu Z, et al. Structural basis of $\mathrm{N}(6)$-adenosine methylation by the METTL3-METTL14 complex. Nature. 2016:534(7608):575-8.

52. Wang Y, Li Y, Toth Jl, Petroski MD, Zhang Z, Zhao JC. N6-methyladenosine modification destabilizes developmental regulators in embryonic stem cells. Nat Cell Biol. 2014;16(2):191-8.

53. Roignant JY, Soller M. m(6)A in mRNA: an ancient mechanism for finetuning gene expression. Trends Genet. 2017;33(6):380-90. 
54. Sledz $P$, Jinek M. Structural insights into the molecular mechanism of the m(6)A writer complex. eLife. 2016. https://doi.org/10.7554/eLife. 18434.

55. Zheng G, Dahl JA, Niu Y, Fedorcsak P, Huang CM, Li CJ, et al. ALKBH5 is a mammalian RNA demethylase that impacts RNA metabolism and mouse fertility. Mol Cell. 2013;49(1):18-29.

56. Jia G, Fu Y, Zhao X, Dai Q, Zheng G, Yang Y, et al. N6-methyladenosine in nuclear RNA is a major substrate of the obesity-associated FTO. Nat Chem Biol. 2011;7(12):885-7.

57. Gerken T, Girard CA, Tung YC, Webby CJ, Saudek V, Hewitson KS, et al. The obesity-associated FTO gene encodes a 2-oxoglutarate-dependent nucleic acid demethylase. Science. 2007;318(5855):1469-72.

58. Zhou B, Han Z. Crystallization and preliminary X-ray diffraction of the RNA demethylase ALKBH5. Acta Crystallogr Sect F Struct Biol Cryst Commun. 2013;69(Pt 11):1231-4.

59. Mauer J, Luo X, Blanjoie A, Jiao X, Grozhik AV, Patil DP, et al. Reversible methylation of m(6)Am in the $5^{\prime}$ cap controls mRNA stability. Nature. 2017;541(7637):371-5

60. Wei J, Liu F, Lu Z, Fei Q, Ai Y, He PC, et al. Differential m(6)A, m(6)Am, and $\mathrm{m}(1) \mathrm{A}$ demethylation mediated by FTO in the cell nucleus and cytoplasm. Mol Cell. 2018;71(6):973-985 e975.

61. Zhang X, Wei LH, Wang Y, Xiao Y, Liu J, Zhang W, et al. Structural insights into FTO's catalytic mechanism for the demethylation of multiple RNA substrates. Proc Natl Acad Sci USA. 2019;116(8):2919-24.

62. Frayling TM, Timpson NJ, Weedon MN, Zeggini E, Freathy RM, Lindgren $\mathrm{CM}$, et al. A common variant in the FTO gene is associated with body mass index and predisposes to childhood and adult obesity. Science. 2007;316(5826):889-94.

63. Shah A, Rashid F, Awan HM, Hu S, Wang X, Chen L, et al. The DEAD-box RNA helicase DDX3 interacts with m(6)A RNA demethylase ALKBH5. Stem Cells Int. 2017;2017:8596135.

64. Zhang X, Lu N, Wang L, Wang Y, Li M, Zhou Y, et al. Recent advances of $\mathrm{m}(6) A$ methylation modification in esophageal squamous cell carcinoma. Cancer Cell Int. 2021;21(1):421.

65. Haussmann IU, Bodi Z, Sanchez-Moran E, Mongan NP, Archer N, Fray $\mathrm{RG}$, et al. m(6)A potentiates Sxl alternative pre-mRNA splicing for robust Drosophila sex determination. Nature. 2016;540(7632):301-4.

66. Batista PJ, Molinie B, Wang J, Qu K, Zhang J, Li L, et al. m(6)A RNA modification controls cell fate transition in mammalian embryonic stem cells. Cell Stem Cell. 2014;15(6):707-19.

67. Geula S, Moshitch-Moshkovitz S, Dominissini D, Mansour AA, Kol N, Salmon-Divon M, et al. m6A mRNA methylation facilitates resolution of naive pluripotency toward differentiation. Science. 2015;347(6225):1002-6.

68. Wang X, Lu Z, Gomez A, Hon GC, Yue Y, Han D, et al. N6-methyladenosine-dependent regulation of messenger RNA stability. Nature. 2014;505(7481):117-20.

69. Wang X, Zhao BS, Roundtree IA, Lu Z, Han D, Ma H, et al. N(6)-methyladenosine modulates messenger RNA translation efficiency. Cell. 2015;161(6):1388-99.

70. Xiao W, Adhikari S, Dahal U, Chen YS, Hao YJ, Sun BF, et al. Nuclear m(6)A reader YTHDC1 regulates mRNA splicing. Mol Cell. 2016;61(4):507-19.

71. Patil DP, Chen CK, Pickering BF, Chow A, Jackson C, Guttman M, et al. m(6)A RNA methylation promotes XIST-mediated transcriptional repression. Nature. 2016;537(7620):369-73.

72. Liu N, Dai Q, Zheng G, He C, Parisien M, Pan T. N(6)-methyladenosinedependent RNA structural switches regulate RNA-protein interactions. Nature. 2015;518(7540):560-4.

73. Dai XY, Shi L, Li Z, Yang HY, Wei JF, Ding Q. Main N6-methyladenosine readers: YTH family proteins in cancers. Front Oncol. 2021;11:635329.

74. Liao S, Sun H, Xu C. YTH domain: a family of N(6)-methyladenosine [m(6)A] readers. Genom Proteom Bioinform. 2018;16(2):99-107.

75. Du H, Zhao Y, He J, Zhang Y, Xi H, Liu M, et al. YTHDF2 destabilizes m(6) A-containing RNA through direct recruitment of the CCR4-NOT deadenylase complex. Nat Commun. 2016;7:12626.

76. Ma Z, Li Q, Liu P, Dong W, Zuo Y. METTL3 regulates m6A in endometrioid epithelial ovarian cancer independently of METTI14 and WTAP. Cell Biol Int. 2020;44(12):2524-31.

77. Huang H, Wang Y, Kandpal M, Zhao G, Cardenas H, Ji Y, et al. FTOdependent N(6)-methyladenosine modifications inhibit ovarian cancer stem cell self-renewal by blocking cAMP signaling. Cancer Res. 2020;80(16):3200-14

78. Fukumoto T, Zhu H, Nacarelli T, Karakashev S, Fatkhutdinov N, Wu S, et al. N(6)-methylation of adenosine of FZD10 mRNA contributes to PARP inhibitor resistance. Cancer Res. 2019;79(11):2812-20.

79. Jiang Y, Wan Y, Gong M, Zhou S, Qiu J, Cheng W. RNA demethylase ALKBH5 promotes ovarian carcinogenesis in a simulated tumour microenvironment through stimulating NF-kappaB pathway. J Cell Mol Med. 2020:24(11):6137-48.

80. Xu F, Li J, Ni M, Cheng J, Zhao H, Wang S, et al. FBW7 suppresses ovarian cancer development by targeting the N(6)-methyladenosine binding protein YTHDF2. Mol Cancer. 2021;20(1):45

81. Muller S, Glass M, Singh AK, Haase J, Bley N, Fuchs T, et al. IGF2BP1 promotes SRF-dependent transcription in cancer in a m6A- and miRNAdependent manner. Nucleic Acids Res. 2019;47(1):375-90.

82. Hao L, Wang JM, Liu BQ, Yan J, Li C, Jiang JY, et al. m6A-YTHDF1-mediated TRIM29 upregulation facilitates the stem cell-like phenotype of cisplatin-resistant ovarian cancer cells. Biochim Biophys Acta Mol Cell Res. 2021;1868(1):118878.

83. Liang S, Guan H, Lin X, Li N, Geng F, Li J. METTL3 serves an oncogenic role in human ovarian cancer cells partially via the AKT signaling pathway. Oncol Lett. 2020;19(4):3197-204.

84. Li X, Tang J, Huang W, Wang F, Li P, Qin C, et al. The M6A methyltransferase METTL3: acting as a tumor suppressor in renal cell carcinoma. Oncotarget. 2017;8(56):96103-16.

85. Zhao S, Liu J, Nanga P, Liu Y, Cicek AE, Knoblauch N, et al. Detailed modeling of positive selection improves detection of cancer driver genes. Nat Commun. 2019;10(1):3399.

86. Ma S, Chen C, Ji X, Liu J, Zhou Q, Wang G, et al. The interplay between m6A RNA methylation and noncoding RNA in cancer. J Hematol Oncol. 2019:12(1):121.

87. Liu J, Yue Y, Han D, Wang X, Fu Y, Zhang L, et al. A METTL3-METTL14 complex mediates mammalian nuclear RNA N6-adenosine methylation. Nat Chem Biol. 2014;10(2):93-5.

88. Shimba S, Bokar JA, Rottman F, Reddy R. Accurate and efficient $\mathrm{N}$-6-adenosine methylation in spliceosomal U6 small nuclear RNA by HeLa cell extract in vitro. Nucleic Acids Res. 1995;23(13):2421-6.

89. Choe J, Lin S, Zhang W, Liu Q, Wang L, Ramirez-Moya J, et al. mRNA circularization by METTL3-eIF3h enhances translation and promotes oncogenesis. Nature. 2018;561(7724):556-60.

90. Lin S, Choe J, Du P, Triboulet R, Gregory RI. The m(6)A methyltransferase METTL3 promotes translation in human cancer cells. Mol Cell. 2016;62(3):335-45.

91. Dong S, Wu Y, Liu Y, Weng H, Huang H. N(6)-methyladenosine steers RNA metabolism and regulation in cancer. Cancer Commun. 2021;41(7):538-59.

92. Singh N, Bhalla N. Moonlighting proteins. Annu Rev Genet. 2020;54:265-85.

93. Zheng QK, Ma C, Ullah I, Hu K, Ma RJ, Zhang N, et al. Roles of N6-methyladenosine demethylase FTO in malignant tumors progression. Onco Targets Ther. 2021;14:4837-46.

94. Wang J, Wang J, Gu Q, Ma Y, Yang Y, Zhu J, et al. The biological function of m6A demethylase ALKBH5 and its role in human disease. Cancer Cell Int. 2020;20:347

95. Zhang Z, Zhu H, Hu J. CircRAB1 1FIP1 promoted autophagy flux of ovarian cancer through DSC1 and miR-129. Cell Death Dis. 2021;12(2):219.

96. Piette ER, Moore JH. Identification of epistatic interactions between the human RNA demethylases FTO and ALKBH5 with gene set enrichment analysis informed by differential methylation. BMC Proc. 2018;12(Suppl 9):59.

97. Hernandez-Caballero ME, Sierra-Ramirez JA. Single nucleotide polymorphisms of the FTO gene and cancer risk: an overview. Mol Biol Rep. 2015;42(3):699-704

98. Kaklamani V, Yi N, Sadim M, Siziopikou K, Zhang K, Xu Y, et al. The role of the fat mass and obesity associated gene (FTO) in breast cancer risk. BMC Med Genet. 2011;12:52.

99. Iles MM, Law MH, Stacey SN, Han J, Fang S, Pfeiffer R, et al. A variant in FTO shows association with melanoma risk not due to BMI. Nat Genet. 2013;45(4):428-32.

100. Kenda Suster N, Virant-Klun I. Presence and role of stem cells in ovarian cancer. World J Stem Cells. 2019:11(7):383-97. 
101. Nowicki A, Kulus M, Wieczorkiewicz M, Pienkowski W, Stefanska K, Skupin-Mrugalska P, et al. Ovarian cancer and cancer stem cells-cellular and molecular characteristics, signaling pathways, and usefulness as a diagnostic tool in medicine and oncology. Cancers. 2021. https://doi. org/10.3390/cancers13164178.

102. Dong P, Xiong Y, Yue J, Hanley SJB, Watari H. Tumor-intrinsic PD-L1 signaling in cancer initiation, development and treatment: beyond immune evasion. Front Oncol. 2018;8:386.

103. Amaravadi RK, Kimmelman AC, Debnath J. Targeting autophagy in cancer: recent advances and future directions. Cancer Discov. 2019;9(9):1167-81.

104. Dikic I, Elazar Z. Mechanism and medical implications of mammalian autophagy. Nat Rev Mol Cell Biol. 2018;19(6):349-64.

105. White E, Lattime EC, Guo JY. Autophagy regulates stress responses, metabolism, and anticancer immunity. Trends Cancer. 2021;7(8):778-89.

106. Kitada M, Koya D. Autophagy in metabolic disease and ageing. Nat Rev Endocrinol. 2021. https://doi.org/10.1038/s41574-021-00551-9.

107. Miller DR, Thorburn A. Autophagy and organelle homeostasis in cancer. Dev Cell. 2021;56(7):906-18

108. Wang Y, Qin C, Yang G, Zhao B, Wang W. The role of autophagy in pancreatic cancer progression. Biochim Biophys Acta Rev Cancer. 2021;1876(2):188592.

109. Ascenzi F, De Vitis C, Maugeri-Sacca M, Napoli C, Ciliberto G, Mancini R. SCD1, autophagy and cancer: implications for therapy. J Exp Clin Cancer Res. 2021:40(1):265.

110. Aventaggiato M, Vernucci E, Barreca F, Russo MA, Tafani M. Sirtuins' control of autophagy and mitophagy in cancer. Pharmacol Ther. 2021;221:107748.

111. Wang X, Wu R, Liu Y, Zhao Y, Bi Z, Yao Y, et al. m(6)A mRNA methylation controls autophagy and adipogenesis by targeting Atg5 and Atg7. Autophagy. 2020;16(7):1221-35.

112. Li J, Wu L, Pei M, Zhang Y. YTHDF2, a protein repressed by miR-145, regulates proliferation, apoptosis, and migration in ovarian cancer cells. J Ovarian Res. 2020;13(1):111.

113. Huang $X$, Zhang $H$, Guo $X$, Zhu Z, Cai H, Kong X. Insulin-like growth factor 2 mRNA-binding protein 1 (IGF2BP1) in cancer. J Hematol Oncol. 2018;11(1):88

114. Jia R, Chai P, Wang S, Sun B, Xu Y, Yang Y, et al. m(6)A modification suppresses ocular melanoma through modulating HINT2 mRNA translation. Mol Cancer. 2019;18(1):161.

115. Shen X, Zhao K, Xu L, Cheng G, Zhu J, Gan L, et al. YTHDF2 inhibits gastric cancer cell growth by regulating FOXC2 signaling pathway. Front Genet. 2020;11:592042.

116. Yang S, Wei J, Cui YH, Park G, Shah P, Deng Y, et al. m(6)A mRNA demethylase FTO regulates melanoma tumorigenicity and response to anti-PD-1 blockade. Nat Commun. 2019;10(1):2782

117. Yang Z, Li J, Feng G, Gao S, Wang Y, Zhang S, et al. MicroRNA-145 modulates N(6)-methyladenosine levels by targeting the $3^{\prime}$-untranslated mRNA region of the N(6)-methyladenosine binding YTH domain family 2 protein. J Biol Chem. 2017;292(9):3614-23.

118. Song $H$, Feng $X$, Zhang H, Luo Y, Huang J, Lin M, et al. METTL3 and ALKBH5 oppositely regulate m(6)A modification of TFEB mRNA, which dictates the fate of hypoxia/reoxygenation-treated cardiomyocytes. Autophagy. 2019;15(8):1419-37.

119. Gao R, Ye M, Liu B, Wei M, Ma D, Dong K. m6A modification: a doubleedged sword in tumor development. Front Oncol. 2021;11:679367.

120. Li J, Han Y, Zhang H, Qian Z, Jia W, Gao Y, et al. The m6A demethylase FTO promotes the growth of lung cancer cells by regulating the m6A level of USP7 mRNA. Biochem Biophys Res Commun. 2019:512(3):479-85

121. Liu J, Ren D, Du Z, Wang H, Zhang H, Jin Y. m(6)A demethylase FTO facilitates tumor progression in lung squamous cell carcinoma by regulating MZF1 expression. Biochem Biophys Res Commun. 2018;502(4):456-64.

122. Du M, Zhang Y, Mao Y, Mou J, Zhao J, Xue Q, et al. MiR-33a suppresses proliferation of NSCLC cells via targeting METTL3 mRNA. Biochem Biophys Res Commun. 2017;482(4):582-9.

123. Wu L, Wu D, Ning J, Liu W, Zhang D. Changes of N6-methyladenosine modulators promote breast cancer progression. BMC Cancer. 2019:19(1):326.
124. Wang H, Xu B, Shi J. N6-methyladenosine METTL3 promotes the breast cancer progression via targeting BCl-2. Gene. 2020;722:144076.

125. $\mathrm{Vu}$ LP, Pickering BF, Cheng $Y$, Zaccara S, Nguyen D, Minuesa G, et al. The N(6)-methyladenosine [m(6)A]-forming enzyme METTL3 controls myeloid differentiation of normal hematopoietic and leukemia cells. Nat Med. 2017;23(11):1369-76.

126. Li Z, Weng H, Su R, Weng X, Zuo Z, Li C, et al. FTO plays an oncogenic role in acute myeloid leukemia as a N(6)-methyladenosine RNA demethylase. Cancer Cell. 2017;31(1):127-41.

127. Cancer Genome Atlas Research N, Ley TJ, Miller C, Ding L, Raphael BJ, Mungall AJ, et al. Genomic and epigenomic landscapes of adult de novo acute myeloid leukemia. N Engl J Med. 2013;368(22):2059-74.

128. Weng H, Huang H, Wu H, Qin X, Zhao BS, Dong L, et al. METTL14 inhibits hematopoietic stem/progenitor differentiation and promotes leukemogenesis via mRNA m(6)A modification. Cell Stem Cell. 2018:22(2):191-205 e199.

129. Bansal H, Yihua Q, lyer SP, Ganapathy S, Proia DA, Penalva LO, et al. WTAP is a novel oncogenic protein in acute myeloid leukemia. Leukemia. 2014;28(5):1171-4.

130. Cui Q, Shi H, Ye P, Li L, Qu Q, Sun G, et al. m(6)A RNA methylation regulates the self-renewal and tumorigenesis of glioblastoma stem cells. Cell Rep. 2017;18(11):2622-34.

131. Visvanathan A, Patil V, Arora A, Hegde AS, Arivazhagan A, Santosh $V$, et al. Essential role of METTL3-mediated m(6)A modification in glioma stem-like cells maintenance and radioresistance. Oncogene. 2018;37(4):522-33

132. Gugnoni M, Sancisi V, Manzotti G, Gandolfi G, Ciarrocchi A. Autophagy and epithelial-mesenchymal transition: an intricate interplay in cancer. Cell Death Dis. 2016;7(12):e2520.

133. Wang Q, Zhang Q, Li Q, Zhang J, Zhang J. Clinicopathological and immunological characterization of RNA m(6)A methylation regulators in ovarian cancer. Mol Genet Genomic Med. 2021;9(1):e1547.

134. Fan L, Lin Y, Lei H, Shu G, He L, Yan Z, et al. A newly defined risk signature, consisting of three m(6)A RNA methylation regulators, predicts the prognosis of ovarian cancer. Aging. 2020;12(18):18453-75.

135. Wei $Q$, Yang $D$, Liu $X$, Zhao $H$, Yang $Y, X u$ J, et al. Exploration of the role of $\mathrm{m}(6) \mathrm{A}$ RNA methylation regulators in malignant progression and clinical prognosis of ovarian cancer. Front Genet. 2021;12:650554.

136. Yang Y, Wei Q, Tang Y, Yuanyuan W, Luo Q, Zhao H, et al. Loss of hnRNPA2B1 inhibits malignant capability and promotes apoptosis via down-regulating Lin28B expression in ovarian cancer. Cancer Lett. 2020:475:43-52

137. Li B, Jiang J, Assaraf YG, Xiao H, Chen ZS, Huang C. Surmounting cancer drug resistance: new insights from the perspective of $N(6)$-methyladenosine RNA modification. Drug Resist Updat. 2020;53:100720.

138. Huang Y, Yan J, Li Q, Li J, Gong S, Zhou H, et al. Meclofenamic acid selectively inhibits FTO demethylation of m6A over ALKBH5. Nucleic Acids Res. 2015;43(1):373-84

139. Peng S, Xiao W, Ju D, Sun B, Hou N, Liu Q, et al. Identification of entacapone as a chemical inhibitor of FTO mediating metabolic regulation through FOXO1. Sci Transl Med. 2019. https://doi.org/10. 1126/scitransImed.aau7116.

140. Huang $Y$, Su R, Sheng $Y$, Dong L, Dong Z, Xu H, et al. Small-Molecule Targeting of Oncogenic FTO Demethylase in Acute Myeloid Leukemia. Cancer Cell. 2019:35(4):677-691 e610.

141. Wagle M, Eiring AM, Wongchenko M, Lu S, Guan Y, Wang Y, et al. A role for FOXO1 in BCR-ABL1-independent tyrosine kinase inhibitor resistance in chronic myeloid leukemia. Leukemia. 2016;30(7):1493-501

142. Kim YJ, Lee SA, Myung SC, Kim W, Lee CS. Radicicol, an inhibitor of Hsp90, enhances TRAIL-induced apoptosis in human epithelial ovarian carcinoma cells by promoting activation of apoptosis-related proteins. Mol Cell Biochem. 2012;359(1-2):33-43.

143. Wang R, Han Z, Liu B, Zhou B, Wang N, Jiang Q, et al. Identification of natural compound radicicol as a potent FTO inhibitor. Mol Pharm. 2018;15(9):4092-8

144. Selberg S, Blokhina D, Aatonen M, Koivisto P, Siltanen A, Mervaala E, et al. Discovery of small molecules that activate RNA methylation through cooperative binding to the METTL3-14-WTAP complex active site. Cell Rep. 2019;26(13):3762-3771 e3765. 
145. Bedi RK, Huang D, Eberle SA, Wiedmer L, Sledz P, Caflisch A. Small-molecule inhibitors of METTL3, the major human epitranscriptomic writer. ChemMedChem. 2020;15(9):744-8.

146. Yankova E, Blackaby W, Albertella M, Rak J, De Braekeleer E, Tsagkogeorga G, et al. Small-molecule inhibition of METTL3 as a strategy against myeloid leukaemia. Nature. 2021;593(7860):597-601.

147. Moroz-Omori EV, Huang D, Kumar Bedi R, Cheriyamkunnel SJ, Bochenkova E, Dolbois A, et al. METTL3 inhibitors for epitranscriptomic modulation of cellular processes. ChemMedChem. 2021;16(19):3035-43.

148. Li J, Gregory Rl. Mining for METTL3 inhibitors to suppress cancer. Nat Struct Mol Biol. 2021;28(6):460-2.

149. Jin J, Wu Y, Chen J, Shen Y, Zhang L, Zhang H, et al. The peptide PROTAC modality: a novel strategy for targeted protein ubiquitination. Theranostics. 2020;10(22):10141-53.

150. Hines J, Gough JD, Corson TW, Crews CM. Posttranslational protein knockdown coupled to receptor tyrosine kinase activation with phosphoPROTACs. Proc Natl Acad Sci USA. 2013;1 10(22):8942-7.

151. Li J, Choi PS, Chaffer CL, Labella K, Hwang JH, Giacomelli AO, et al. An alternative splicing switch in FLNB promotes the mesenchymal cell state in human breast cancer. eLife. 2018. https://doi.org/10.7554/eLife. 37184.

152. Bechara EG, Sebestyen E, Bernardis I, Eyras E, Valcarcel J. RBM5, 6, and 10 differentially regulate NUMB alternative splicing to control cancer cell proliferation. Mol Cell. 2013;52(5):720-33.

153. Gokmen-Polar Y, Neelamraju Y, Goswami CP, Gu Y, Gu X, Nallamothu G, et al. Splicing factor ESRP1 controls ER-positive breast cancer by altering metabolic pathways. EMBO Rep. 2019. https://doi.org/10.15252/embr. 201846078

154. Wang Y, Chen D, Qian H, Tsai YS, Shao S, Liu Q, et al. The splicing factor RBM4 controls apoptosis, proliferation, and migration to suppress tumor progression. Cancer Cell. 2014:26(3):374-89.

155. Tripathi V, Shin JH, Stuelten CH, Zhang YE. TGF-beta-induced alternative splicing of TAK1 promotes EMT and drug resistance. Oncogene. 2019;38(17):3185-200

156. Wang Y, Bernhardy AJ, Cruz C, Krais JJ, Nacson J, Nicolas E, et al. The BRCA1-Delta1 1q alternative splice isoform bypasses germline mutations and promotes therapeutic resistance to PARP inhibition and cisplatin. Cancer Res. 2016;76(9):2778-90

157. Tang C, Klukovich R, Peng H, Wang Z, Yu T, Zhang Y, et al. ALKBH5dependent m6A demethylation controls splicing and stability of long 3'-UTR mRNAs in male germ cells. Proc Natl Acad Sci USA. 2018;115(2):E325-33.

158. Paul BT, Blanchard Z, Ridgway M, ElShamy WM. BRCA1-IRIS inactivation sensitizes ovarian tumors to cisplatin. Oncogene. 2015;34(23):3036-52.

159. Desterro J, Bak-Gordon P, Carmo-Fonseca M. Targeting mRNA processing as an anticancer strategy. Nat Rev Drug Discov. 2020;19(2):112-29.

160. Crooke ST, Witztum JL, Bennett CF, Baker BF. RNA-targeted therapeutics. Cell Metab. 2018;27(4):714-39.

\section{Publisher's Note}

Springer Nature remains neutral with regard to jurisdictional claims in published maps and institutional affiliations.

Ready to submit your research? Choose BMC and benefit from:

- fast, convenient online submission

- thorough peer review by experienced researchers in your field

- rapid publication on acceptance

- support for research data, including large and complex data types

- gold Open Access which fosters wider collaboration and increased citations

- maximum visibility for your research: over $100 \mathrm{M}$ website views per year

At $\mathrm{BMC}$, research is always in progress.

Learn more biomedcentral.com/submissions 